\title{
Health is Wealth \& Wealth is Pugos Nutrition
}

\author{
Dr.Govind Shukla \& C.J.Sampath Kumar
}

PUGOS Products Pvt. Ltd., Leelavathi Mansion, Margosa Main Road, Malleshwaram, Bangalore-56003, India.

\section{ABSTRACT}

Hippocrates wisely stated back in $400 \mathrm{BC}$, "Let food be your medicine and medicine be your food". Modern research has validated this doctrine. The science of nutrition is dedicated to learning about foods that the human body requires at different stages of life in order to meet the nutritional needs for proper growth, as well as to maintain health and prevent disease. A baby is born with a very high requirement for energy and nutrient intake per unit of body weight to provide for rapid growth. The rate of growth is the highest during the first year and declines slowly after the age of two, with a corresponding decrease in nutrient and energy requirements. During puberty, however, nutritional requirements increase sharply until this period of fast growth is completed. Adulthood begins at about the age of fourteen or fifteen for girls, and eighteen or nineteen for boys.

An adult individual needs to balance energy intake with his or her level of physical activity to avoid storing excess body fat. Dietary practices and food choices are related to wellness and affect health, fitness, weight management, and the prevention of chronic diseases such as osteoporosis, cardiovascular diseases, cancer, and diabetes.

Nutrition describes the processes by which all of the food a person eats are taken in and the nutrients that the body needs are absorbed. Good nutrition like Pugos Nutrition can help prevent disease and promote health.

\section{Introduction}

Macronutrients are essential nutrients - carbohydrates, proteins, and fats - that the body needs for energy and proper growth, metabolism, and function. They are called "macro" because we need these nutrients in large quantities compared to the micronutrients (vitamins and minerals), which are needed in smaller quantities.

\section{Protein}

Protein is a necessary component for building, maintenance, and repair of many body systems and processes, including:

- Production of collagen and keratin, which are the structural components of bones, teeth, hair, and the outer layer of skin; they help maintain the structure of blood vessels.

- Manufacture of hormones, such as insulin and thyroid hormone.

- Production of enzymes that control chemical reactions in the body.

- Proper immune function — production of antibodies, white blood cells, and other immune factors.

- Transportation of oxygen, vitamins, and minerals to target cells throughout the body.

\section{Food Sources}

Protein is found in animal products, nuts, legumes, and, to a lesser extent, in fruits and vegetables. When we eat protein the body breaks it down into amino acids, some of which are called essential because they must be provided by the food we eat. Others that can be produced by the body are called non-essential.

Protein from animal sources contains all of the essential amino acids. Therefore, best sources of lean protein are chicken, turkey, fish, and eggs. Plant proteins do not contain all the essential amino acids and are considered 
incomplete proteins. It is possible, though, to combine various plant proteins to get all the essential amino acids. For example, eating oats, lentils, and sunflower seeds either together or separately throughout the day provides all the essential amino acids.

A number of studies have found lower risk of chronic disease in those who eat a plant-based diet. The National Institute of Nutrition recommends ranges for macronutrient intake that are associated with a reduced risk of chronic disease while providing adequate intake of essential nutrients. They suggest that adults get $45-65$ percent of calories from carbohydrates, 20-35 percent from fat, and 10-35 percent from protein. Ranges for children are similar, except that infants and younger children need a slightly higher proportion of fat (25-40 percent).

\section{Carbohydrates}

Carbohydrates are the body's main source of fuel—glucose, which is needed by every cell in our body. They also provide valuable nutrients (vitamins, minerals, and essential fatty acids) and fibre, which is important for intestinal health.

\section{Food Sources}

There are two classes of carbohydrates - simple and complex. Simple carbohydrates include naturally occurring sugars in milk and fruit, and refined sugars (granulated sugar). There is a major difference among these simple carbohydrates: fruits offer a range of nutrients and fibre, while refined sugars provide empty calories and lack nutritional value. Excess sugar consumption is linked to dental caries, obesity, insulin resistance, high triglycerides, low HDL (good) cholesterol, and compromised immune function. The World Health Organization recommends reducing sugar intake to below 10 percent of total calories. Aside from candy and baked goods, sugar is also found in pop, condiments (ketchup, barbecue sauces), juices, ice cream, and other sweets.

Complex carbohydrates include starches and indigestible dietary fibre. Starches are found in bread, pasta, rice, beans, and some vegetables. Today many of our starches are refined and processed, which strips the food of its fibre and nutrients. For example, white bread, pasta, and rice are much less nutritious, so choose the brown or whole-grain products. Dietary fibre is found in fruits, vegetables, beans, and the indigestible parts of whole grains such as wheat and oat bran. In addition to supporting intestinal health and proper elimination, fibre also improves blood sugar balance, lowers cholesterol, reduces the risk of colon and breast cancer, and plays a role in weight management. The recommended intake of fibre for adults 50 years and younger is $38 \mathrm{~g}$ for men and $25 \mathrm{~g}$ for women; for men and women over 50 it is 30 and $21 \mathrm{~g}$ per day, respectively, due to decreased food consumption. Sadly, most people get only one third to one-half of the recommended amount. To boost fibre intake, incorporate more raw vegetables, fruits, whole grains, and legumes in your diet and consider a fibre supplement.

\section{Glycemic Index}

The glycemic index (GI) is a scale that measures how quickly carbohydrates are broken down into sugar. Those that are broken down quickly_-such as simple carbohydrates and refined starches-have a high GI. Foods that are broken down slowly—such as most vegetables, fruits, and unprocessed grains- have a low GI. Numerous studies have linked high-GI diets to obesity, insulin resistance, type 2 diabetes, and increased risk of heart disease. Eating 
Asian Journal of Applied Science and Technology

Volume 4, Issue 3, Pages 215-284, July-September 2020

high-GI foods can lead to blood sugar imbalances that may result in fatigue, increased appetite, and food cravings. For these reasons, it is best to minimize high-GI foods and maximize your intake of low-GI foods.

\section{Fats}

"Fat" has become a negative word as it is associated with obesity, yet we do need a certain amount of fat in our diets and on our bodies. The point to keep in mind is that there are good fats and bad fats.

The good fats are the unsaturated fats, namely, the monounsaturated fats (olive, canola, and peanut oil) and polyunsaturated fats. The polyunsaturated fats provide us with essential fatty acids (EFAs), which are broken down into two groups:

- Omega-6 fatty acids: Linoleic acid (LA), which is converted into gamma-linolenic acid (GLA) and arachidonic acid (AA)

- Omega-3 fatty acids: Alpha-linolenic acid (ALA), which is converted into eicosapentaenoic acid (EPA) and docosahexaenoic acid (DHA)

The body cannot make EFAs, so they must be obtained through diet or supplementation. They are essential for many body processes and functions, including:

- Growth and development of brain, nervous system, adrenal glands, sex organs, inner ear, and eyes

- Energy (fat is the most concentrated source of energy)

- Absorbing fat-soluble vitamins (vitamins A, D, E, K, and carotenoids)

- Maintaining cell membrane integrity

- Regulation of cell processes such as gene activation and expression, enzyme function, and fat oxidation

- Production of hormones and chemical messengers

\section{Food Sources}

Here is a breakdown of the EFAs and their sources:

- LA: Found in vegetable oils such as safflower, evening primrose, sunflower, corn, hemp, canola, and olive oil.

- GLA: Found in borage, blackcurrant, and evening primrose oils.

- $A A$ : Found in meat and eggs. We get adequate AA through diet. Too much of this fat is not good, as it causes inflammation.

- $A L A$ : Found in flaxseed and hemp oil and, to a lesser extent, in nuts, green leafy vegetables, wheat germ, and blackcurrant seeds.

- EPA and DHA: Found in fatty fish, such as salmon, mackerel, herring, cod, sardines, and tuna. There is great controversy over what constitutes the optimal dietary intake ratio of omega- 6 to omega- 3 fatty acids. It is estimated that we currently get around 15:1, whereas leading EFA authorities recommend a ratio closer to 4:1 or even 2:1. 
The Institute of Medicine has set an adequate intake level for linoleic acid for adults 19-50 years of age at $17 \mathrm{~g} /$ day for men and $12 \mathrm{~g} /$ day for women; alpha-linolenic acid at $1.6 \mathrm{~g} /$ day for men and $1.1 \mathrm{~g} / \mathrm{day}$ for women. These levels are lower for younger and older individuals.

Aim to have more omega-3s (fish, flaxseed, hemp, and fish oils) and GLA (borage, blackcurrant, or primrose oil) from diet and/or supplements, as these are the beneficial fats that are commonly deficient. Diets rich in the omega-3 fatty acids offer cardio protection by lowering blood cholesterol and triglyceride levels, reducing blood clotting, and reducing the risk of heart attack and sudden death. These fats also reduce inflammation and are helpful for arthritis and other inflammatory disorders. GLA also reduces inflammation, and prevents clotting, dilates blood vessels, improves skin health, and benefits those with diabetes and arthritis.

\section{Saturated Fats}

Saturated fats are found in animal products such as meat, poultry, milk, cheese, butter, and lard, as well as in tropical oils (such as palm, palm kernel, and coconut oil) and foods made from these oils. These fats are high in cholesterol and linked to heart disease, high cholesterol, obesity, and cancers of the breast, colon, and prostate.

Most people get 38 percent or more of the day's calories from fat while health authorities suggest no more than 20-35 percent of which less than 10 percent comes from saturated fat.

\section{Trans Fats}

Trans fatty acids are naturally found in small amounts in animal products; however, the majority of trans fats in our diet come from the artificial form. Trans fats are created when oils undergo a chemical process called hydrogenation, which solidifies them. This is the process that makes vegetable oil into margarine. Trans fat is also found in cookies, crackers, french fries, baked goods, and other snack foods.

When trans fats were first introduced into our food supply, they were thought to be a healthier alternative to saturated fats. Many years later this was found to be false. Trans fats elevate cholesterol levels, increasing the risk for heart disease and heart attack, and are also linked to cancer, particularly breast cancer.

\section{Cholesterol}

Cholesterol is a waxy substance found in the fats (lipids) in our blood. It is manufactured in the liver and also obtained from consuming saturated and trans fats.

Cholesterol is not all bad - the body requires it to produce sex hormones, maintain cell membranes, and for a healthy nervous system.

Aside from diet, cholesterol levels can be elevated by family history, lack of activity, and liver disorders, and cholesterol consumption increases the risk of heart disease.

As with fats, there is good and bad when it comes to cholesterol. The good cholesterol is HDL (high-density lipoproteins) and the bad is LDL (low-density lipoproteins). LDL cholesterol can build up in the artery walls of the brain and heart, narrowing the passageways for blood flow, a process known as atherosclerosis, the precursor to 
heart disease and stroke. HDL cholesterol is called good cholesterol because it picks up the LDL deposited in the arteries and transports it to the liver to be broken down and eliminated. To lower LDL and raise HDL levels, exercise regularly, minimize saturated fats, avoid trans fats, and don't smoke (smoking lowers HDL).

\section{Triglycerides}

Triglycerides (TG) are the chemical form in which most fats exist in food (both animal and plant fats). They are also present in the blood along with cholesterol.

A diet that is high in fat, sugar, refined carbohydrates, and alcohol can elevate TGs. Overeating also raises TG because excess calories are converted to fat in the liver and then into TG to be transported in the blood. High levels of triglycerides are associated with heart disease and diabetes. It is possible for triglycerides to be high even when blood cholesterol is normal, so get your levels checked regularly. In most cases, TG levels can be effectively managed with diet and exercise.

Micronutrients are vitamins and minerals nutrients required by the body in small amounts yet have powerful effects. They assist in energy-producing reactions, growth and development, protect against free radical damage, and perform many vital functions. Micronutrients are essential for health, and a deficiency can lead to health problems and disease.

National Institute of Nutrition released a report providing reference values for nutrient intakes including:

- Recommended Dietary Allowance (RDA): The average daily dietary nutrient intake level sufficient to meet the nutrient requirement of nearly all (97-98 percent) healthy individuals in a particular life stage and gender group.

- Adequate Intake (AI): The recommended average daily intake level based on observed or experimentally determined estimates of nutrient intake of apparently healthy people that are assumed to be adequate. The AI is given when an RDA cannot be determined.

- Tolerable Upper Intake Level (UL): The highest average daily nutrient intake level that is likely to pose no risk of adverse health effects for almost all individuals in the general population. As intake increases above the UL, the potential risk of adverse effects may increase.

\section{Who Needs Supplements?}

There are many factors that cause nutrient depletion, such as poor diet, stress, exercise, use of prescription drugs, environmental toxicity, and excessive alcohol intake. For many micronutrients, deficiency, inadequate intake or nutrient depletion is common relative to the RDA. This is why supplements are so important in making up for shortcomings in the diet and preventing deficiencies.

\section{Vitamins}

There are 13 essential vitamins that our bodies need for proper growth, function, and maintenance of healthy tissues. The vitamins are either water-soluble or fat-soluble. The B-vitamins and vitamin C dissolve in water and are easily eliminated from the body. Adverse reactions, even with high-dose supplements, are rare with these 
Asian Journal of Applied Science and Technology Volume 4, Issue 3, Pages 215-284, July-September 2020

vitamins. Fat-soluble vitamins (A, D, and E) are not readily excreted from the body and have the potential to accumulate in the tissues and cause adverse effects at high doses.

\section{Vitamin A}

- Found in animal foods and converted from beta-carotene in plant foods.

- Required for vision, gene expression, reproduction, embryonic development, red blood cell production, and immune function.

- Prescription vitamin A derivatives are used to treat skin conditions (acne) and retinitis pigmentosa (genetic eye disease).

- Deficiency is common in developing countries due to malnutrition. It causes night blindness, dry eyes and skin, and impaired growth.

- Drugs that deplete vitamin A: cholestyramine, colestipol, mineral oil, and neomycin.

- Supplements should be avoided by those at risk of lung cancer (smokers) or liver toxicity (alcoholics, liver disease).

- Doses greater than 10,000 IU daily should be avoided by pregnant women due to the risk of birth defects. Most prenatal vitamins provide 5,000 IU.

- Doses greater than 5,000 IU may increase risk of osteoporosis.

- Supplements of vitamin A beyond what is provided in a multivitamin are not recommended due to risk of toxicity. To avoid this risk, choose a multivitamin that contains beta-carotene, which is converted to vitamin $\mathrm{A}$ in the liver, but is not associated with health risks.

\section{Vitamin B1 (Thiamine)}

- Required for energy production, nerve and muscle function, enzyme reactions, and fatty acid production.

- Deficiency causes beriberi, a disease that affects cardiovascular, nervous, muscular, and gastrointestinal systems.

- Deficiency is common in developing countries; in North America it occurs in alcoholics, those with kidney disease, malabsorption syndromes (celiac disease), and in those with poor diets.

- Drugs that deplete vitamin B1: furosemide, antibiotics, oral contraceptives, and phenytoin.

- Most people get adequate thiamine from diet and/or a multivitamin.

\section{Vitamin B2 (Riboflavin)}

- Required for energy metabolism, enzyme reactions, vision, and skin/hair/nail health; functions as an antioxidant; activates vitamin B6, niacin, and folate.

- May play a role in preventing migraine headaches and cataracts.

- Deficiency occurs in alcoholics, the elderly, and those with poor diets. 
- Symptoms of deficiency include sore throat; redness/swelling of the mouth, throat, tongue, lips, and skin; decreased red blood cell count; and blood vessel growth over the eyes. Deficiency may impair iron absorption and increase risk of pre-eclampsia in pregnant women.

- Drugs that deplete vitamin B2: antibiotics, chlorpromazine, amitriptyline, adriamycin, and phenobarbitol.

- Most people get adequate riboflavin from diet and/or a multivitamin.

\section{Vitamin B3 (Niacin)}

- Required for energy metabolism, enzyme reactions, skin and nerve health, and digestion.

- High doses of nicotinic acid (3 g daily) can lower cholesterol (reduce LDL and triglycerides and increase HDL) and reduce the risk of heart attack and stroke; high dosages should be supervised by a physician.

- Deficiency causes pellagra, the symptoms of which are skin rash, diarrhea, dementia, and death.

- Deficiency may be caused by poor diet, malabsorption diseases, dialysis, and HIV.

- Drugs that deplete vitamin B3: antibiotics, isoniazid, and 5-Fluorouracil (chemotherapy).

- High-dose niacin, taken along with statin drugs (i.e., lovastatin), may increase the risk of rhabdomyolysis (muscle degeneration and kidney disease).

- Most people get adequate niacin from diet and/or a multivitamin; supplements may be recommended for those with high cholesterol.

\section{Vitamin B5 (Pantothenic Acid)}

- Required for carbohydrate metabolism, adrenal function, enzyme reactions, and production of fats, cholesterol, bile acids, hormones, neurotransmitters, and red blood cells.

- Deficiency is rare, except in malnutrition, and causes burning/tingling in hands and feet, fatigue, and headache.

- Drugs that deplete vitamin B5: oral contraceptives, amitriptyline, imipramine, and desipramine.

- Most people get adequate niacin from diet and/or a multivitamin.

\section{Vitamin B6 (Pyridoxine)}

- Necessary for protein and fat metabolism, hormone function (estrogen and testosterone), and the production of red blood cells, niacin, and neurotransmitters (serotonin, dopamine, and norepinephrine).

- Used therapeutically for PMS, depression, morning sickness, carpal tunnel syndrome, and heart health (lowers homocysteine, an amino acid that, at high levels, can cause arteriosclerosis and build up arterial plaque).

- Deficiency is uncommon, except in alcoholics and the elderly, and causes seizures, irritability, depression, confusion, mouth sores, and impaired immune function.

- Drugs that deplete vitamin B6: antibiotics, oral contraceptives, isoniazid, penicillamine, and Parkinson's drugs.

- Supplements are recommended for the elderly, alcoholics, and those with poor diets. 
Vitamin B12 (Cobalamin)

- Required for nerve function, synthesis of DNA and RNA, metabolism of energy, enzyme reactions, and production of red blood cells.

- Used therapeutically for heart health (lowers homocysteine), male infertility, prevention of neural tube defects, asthma, and cancer prevention.

- Deficiency is common among the elderly and those with poor diets, pernicious anemia, depression, Alzheimer's, or malabsorption conditions (celiac disease).

- Deficiency symptoms: anemia, appetite loss, constipation, numbness and tingling in the extremities, and confusion. Pregnant women with deficiency have increased risk of giving birth to a child with neural tube defects.

- Drugs that deplete B12: acid-lowering drugs (omeprazole, lansoprazole, ranitidine), oral contraceptives, antibiotics, cholestyramine, and metformin.

- Supplements are recommended for those over age 50, vegetarians, women planning to become pregnant, those with poor diets, and those at risk of heart disease.

\section{Biotin}

- Part of the B-vitamin family; involved in the synthesis of fat, glycogen, and amino acids and enzyme reactions; required for DNA replication; important for healthy hair and nails.

- Used therapeutically to strengthen fingernails.

- Deficiency is rare except in those with hereditary disorders of biotin metabolism, liver disease, and during pregnancy (due to increased needs). It can also occur in those who consume raw egg white for prolonged periods (weeks to years) because a protein found in egg white (avidin) binds biotin and prevents its absorption or in those given intravenous feeding without biotin supplementation.

- Deficiency symptoms include hair loss; scaly red rash around the eyes, nose, mouth, and genital area; depression; lethargy; hallucination; numbness and tingling of the extremities; and impaired glucose utilization and immune system function.

- Drugs that deplete biotin: primidone, carbamazepine, phenobarbital, phentyoin, valproic acid, and antibiotics.

- Most people get adequate biotin from diet and/or supplements.

\section{Folate (Folic Acid)}

- Part of the B-vitamin family; known as folate when it occurs in foods, or as folic acid when present in supplements or added to foods.

- Required for cell division, growth, amino acid metabolism, enzyme reactions, and production of RNA, DNA, and red blood cells.

- Used for heart health (lowers homocysteine) and prevention of cancer (colon and cervical) and birth defects (neural tube). 
- Deficiency occurs in alcoholics and those with poor diets, and causes anemia, fatigue, weakness, headache, hair loss, diarrhea, and poor immune function. Pregnancy or cancer results in increased rates of cell division and metabolism, increasing the need for folate.

- Drugs that deplete folate: non-steroidal anti-inflammatory drugs (NSAIDs) such as ibuprofen and aspirin, phenytoin, methotrexate phenobarbital, cholestyramine, colestipol, trimethoprim, and sulfasalazine.

- Supplements are recommended for most adults for heart and cancer protection, and especially for pregnant women; multivitamins typically provide the recommended amount of $400 \mathrm{mcg}$ per day.

\section{Vitamin C (Ascorbic Acid)}

- Required for synthesis of collagen (structural component of blood vessels, tendons, and bone), norepinephrine (neurotransmitter), and carnitine (amino acid involved in energy production); promotes wound healing; supports immune function and gum health; and has antioxidant properties.

- Used to prevent cataracts, macular degeneration, heart disease, stroke, cancer, and colds; improve wound healing and response to stress; reduce bronchial spasms in asthmatics; and prevent lead toxicity.

- Severe deficiency causes scurvy (bleeding, bruising, hair and tooth loss, joint pain, and swelling).

- Marginal deficiencies are common among the elderly, alcoholics, and those with cancer, chronic illness, or stress. Symptoms include fatigue, easy bruising, poor wound healing and appetite, anemia, and sore joints.

- Drugs that deplete vitamin C: oral contraceptives, aspirin, corticosteroids, and furosemide.

- Large doses of vitamin C (greater than 1,000 mg/day) may reduce the effect of warfarin (blood-thinning drug).

- The NIN recommends $400 \mathrm{mg}$ of vitamin C daily, which is higher than the RDA, yet much lower than the UL. Most multivitamin supplements provide $60 \mathrm{mg}$ of vitamin C.

- Natural and synthetic forms are chemically identical and have the same effects on the body.

- Mineral salts of ascorbic acid (i.e., calcium ascorbate) are buffered and therefore less acidic and less likely to cause upset stomach.

\section{Vitamin D}

- Regulates calcium and phosphorus levels and promotes absorption of these minerals for growth of bones and teeth; involved in insulin secretion; supports immune function; regulates blood pressure.

- Vitamin D can be produced in the skin upon exposure to sunlight or must be obtained from the diet.

- Used to prevent and treat osteoporosis, psoriasis, autoimmune disease, and to reduce the risk of cancer.

- Deficiency occurs with inadequate dietary intake, limited sun exposure, kidney or liver disease, and alcoholism.

Elderly, dark-skinned, obese people, or those with inflammatory bowel disease and fat-malabsorption syndromes (celiac disease and cystic fibrosis) are also at greater risk. 
- Deficiency causes rickets (weak, deformed bones) in children, osteomalacia (soft bones) and osteoporosis in adults, dental problems, muscle weakness, and tooth decay.

- Drugs that deplete vitamin D: carbamazepine, phenytoin, phenobarbital, cimetidine, ranitidine, cholestyramine, colestipol, orlistat, and mineral oil.

- Since vitamin D is found in few foods and at low amounts, a supplement is recommended for most people. Most multivitamins provide $400 \mathrm{IU}(10 \mathrm{mcg})$. Those with limited sun exposure, osteoporosis, multiple sclerosis, psoriasis, and those over age 65 should consider additional vitamin D.

\section{Vitamin E}

- Is an antioxidant (protects cell membranes against oxidative damage; prevents LDL oxidation) that supports immune function, prevents blood clotting, and dilates blood vessels.

- Used to prevent and treat heart disease, cancer, macular degeneration, and cataracts, enhance immune response, reduce oxidative stress, and improve cognitive function.

- Deficiency is rare, except in those who are malnourished or who have fat-malabsorption conditions (celiac disease, cystic fibrosis); however, suboptimal intake is common and associated with increased risk of heart disease.

- Symptoms of deficiency include impaired balance and coordination, damage to sensory nerves (peripheral neuropathy), muscle weakness (myopathy), and damage to the retina of the eye (pigmented retinopathy).

- Drugs that deplete vitamin E: cholestyramine, colestipol, isoniazid, mineral oil, orlistat, sucralfate, phenobarbitol, phenytoin, and carbamazepine.

- Vitamin E may enhance the blood-thinning effects of warfarin.

- It is difficult to achieve the RDA from diet alone; supplements are particularly necessary to achieve amounts needed for disease prevention.

- Look for natural vitamin E (alpha-tocopherol); the synthetic form ( $d l$-alphatocopherol) is less bioavailable (i.e., less absorbable) and only half as potent.

\section{Vitamin K}

- Essential for blood clotting; required for bone formation and cell growth.

- Useful in the prevention of osteoporosis.

- Deficiency is rare in adults and causes impaired blood clotting; it is more common in newborns and can cause severe bleeding. Injections of vitamin K1 are typically given to newborns.

- Symptoms of deficiency include easy bruising and bleeding (nosebleeds, bleeding gums, blood in the urine or stool, or heavy menstrual bleeding).

- Drugs that deplete: antibiotics, aspirin, phenytoin, phenobarb, cholestyramine, colestipol, orlistat, and mineral oil. 
Asian Journal of Applied Science and Technology

Volume 4, Issue 3, Pages 215-284, July-September 2020

- High intake of vitamin K from foods or supplements can reduce the efficacy of blood-thinning drugs (warfarin).

- Most people get adequate vitamin $\mathrm{K}$ from the diet and/or a multivitamin.

\section{Minerals, Trace Minerals, and Electrolytes}

Minerals are elements that originate in the earth. The body cannot make minerals so they must be obtained through the diet and/or supplements. Both plant and animal foods provide minerals. Plants obtain minerals from the soil they are grown in. Animals get minerals from the plants they eat, and then we get these minerals indirectly by eating the animal products. There are also some minerals present in drinking water, but this varies with geographic location, as does the mineral content of plants.

Minerals are categorized according to our daily requirements. Calcium, magnesium, and phosphorus are considered major minerals since we require a substantial amount of these for health and wellness. The trace minerals, which are required in smaller amounts, are chromium, copper, fluoride, iodine, iron, manganese, molybdenum, selenium, and zinc. The minerals potassium and sodium are known as electrolytes, substances that dissociate into ions (charged particles) in solution, making them capable of conducting electricity.

\section{Minerals}

\section{Calcium}

- The most abundant mineral in the body; essential for building and maintaining bones and teeth; required for muscle contractions and nerve function; regulates blood pressure, blood vessel contractions, and clotting; involved in enzyme reactions. Used for the prevention of osteoporosis, colorectal cancer, pre-eclampsia, and lead toxicity and the treatment of high blood pressure.

- Deficiency can occur with poor diet, abnormal parathyroid function (gland that regulates blood calcium levels), kidney failure, and vitamin D or magnesium deficiency. Symptoms of deficiency include bone loss and weakening, muscle cramps, heart palpitations, tooth decay, back and leg pain, insomnia, nervous disorders, and rickets (bone deformities in children).

- Drugs that deplete calcium: cimetidine, ranitidine, omeprazole, aluminum antacids, corticosteroids, cholestyramine, mineral oil, phenytoin, and furosemide.

- A high intake of sodium (salt), protein, phosphorus (soft drinks and food additives), or caffeine (more than 2 cups of coffee or $300 \mathrm{mg}$ caffeine per day) can promote calcium losses.

- Calcium supplements may reduce the efficacy of calcium channel blockers (drugs used to lower blood pressure); use with thiazide diuretics increases the risk of hypercalcemia (high blood calcium levels); calcium supplements may reduce absorption of antibiotics (tetracycline, quinolones), bisphosphonates (osteoporosis drugs), and levothyroxine (thyroid hormone). It is difficult to meet the RDA through diet alone unless dairy intake is high. Most multivitamin/mineral supplements provide a small amount of calcium because it is quite bulky. Therefore, a separate calcium supplement may be necessary, especially for those at risk of osteoporosis and those with high blood pressure. 
- There are several forms of calcium: Carbonate provides the highest amount of calcium (40 percent) and is inexpensive; citrate provides 21 percent calcium, but may be better absorbed in the elderly and those taking acid-lowering drugs.

- To maximize absorption, take no more than $500 \mathrm{mg}$ of elemental calcium at one time, take with meals, and ensure adequate vitamin $\mathrm{D}$ intake (as this is required for calcium absorption).

- Separate calcium-rich foods and supplements by two hours from iron supplements (calcium reduces iron absorption); avoid drinking tea with meals, as the tannins in tea reduce calcium absorption.

- Some vegetables contain chemicals that inhibit the absorption of calcium, such as oxalic acid, which is found in raw spinach, rhubarb, sweet potato, and dried beans. Cooking these foods releases calcium that is bound to oxalic acid, thus improving the amount you can absorb. Phytic acid, which is found in wheat bran or dried beans, also reduces calcium absorption.

\section{Magnesium}

- Required for nerve and muscle function, formation of bones and teeth, synthesis of the antioxidant glutathione, cell membranes, and body temperature regulation; involved in energy production, numerous enzyme reactions, and synthesis of DNA and RNA. Used to prevent heart disease and in the treatment of high blood pressure, pre-eclampsia, heart disease, diabetes, osteoporosis, migraine headaches, and asthma.

- Deficiency is uncommon, but may occur in those with poor diets, malabsorption syndromes (celiac disease), Crohn's disease, intestinal surgery or inflammation, kidney disease, diabetes, alcoholism, and in the elderly due to reduced absorption. Marginal deficiency (consuming less than the RDA) is common and is estimated to affect 75 percent of people.

- Symptoms of deficiency: muscle cramps and spasms, weakness, insomnia, poor appetite, kidney stones, osteoporosis, nervousness, irritability, anxiety, depression, and high blood pressure.

- Drugs that deplete magnesium: furosemide, hydrochlorothiazine, cholestyramine, and oral contraceptives.

\section{Micronutrients}

- Other interactions: Magnesium reduces absorption of digoxin, nitrofurantoin, antimalarial drugs, quinolone antibiotics, tetracycline, chlorpromazine, alendronate, and etidronate, so separate intake of magnesium from these foods by two hours.

- High doses of zinc (greater than $140 \mathrm{mg} /$ day) reduce magnesium absorption.

- It is difficult to meet the RDA through diet alone; therefore, a multivitamin/mineral supplement is recommended. Certain individuals may require an additional magnesium supplement.

\section{Phosphorus}

- Required for structure of bones, teeth, soft tissue, and cell membranes (phospholipids); energy production and storage; enzyme reactions; hormones; formation of DNA and RNA; and maintaining acid-base balance. 
- Deficiency is rare except among alcoholics and those with kidney disease, malabsorption syndromes (celiac or Crohn's disease), or poor diets.

- Symptoms of deficiency: poor appetite, anemia, muscle weakness, bone pain, rickets in children, osteomalacia in adults, increased risk of infection, and numbness and tingling of extremities.

- Drugs that deplete phosphorus: aluminum and magnesium (antacids and supplements), cholestyramine, and digoxin.

- Most people get adequate phosphorus through diet; supplements are rarely necessary.

\section{Trace Minerals}

\section{Chromium}

-Involved in glucose metabolism (enhances effect of insulin) and enzyme reactions.

- Used for diabetes and for those with impaired glucose tolerance and to lower cholesterol and triglycerides.

- Severe deficiency is rare, but marginal deficiency is common; it is estimated that 90 percent of adults consume less than the RDA.

- The main cause of deficiency is poor dietary intake (high-sugar diets increase urinary excretion of chromium).

- Deficiency results in impaired glucose utilization and may be a contributing factor to the development of type 2 diabetes; symptoms include elevated blood sugar, numbness, and tingling in the extremities and nerve problems.

- Drugs that deplete chromium: corticosteroids (prednisone). Other interactions: Chromium may enhance the blood sugar-lowering effects of insulin and oral drugs (glyburide and metformin), thus requiring a dosage adjustment.

- Since marginal deficiencies are common, a multivitamin/mineral complex containing chromium is recommended. Chromium is available in several forms. Most studies involving chromium were done with the picolinate form, which is readily absorbed and utilized by the body. Certain individuals (diabetics and those at risk for diabetes) may require an additional supplement.

\section{Copper}

- A component of enzymes, which are required for energy production, connective tissue formation, iron metabolism, brain and nervous system, synthesis of neurotransmitters, melanin, myelin, hemoglobin, and the antioxidant superoxide dismutase; involved in regulating gene expression.

- Severe deficiency is rare, but marginal deficiencies are common. The typical diet provides about 50 percent of the RDA. Others at risk: Premature and low birthweight infants with diarrhea; infants fed only cow's milk formula, which is low in copper; those with malnutrition, malabsorption syndromes (celiac disease), cystic fibrosis, and those receiving intravenous feeding.

- Deficiency leads to iron deficiency and anemia, low white blood cell count (increased risk of infection), osteoporosis, loss of skin pigment, and impaired growth in children. 
- Drugs that deplete copper: penicillamine, ethambutol, and zidovudine.

- Other interactions: Prolonged high doses of zinc (50 mg daily or more) may result in copper deficiency.

- A varied diet provides adequate copper for most individuals. In addition, taking a multivitamin/mineral complex will provide the RDA.

\section{Fluoride}

- Essential for formation of healthy bones and teeth.

- Used to prevent cavities, harden tooth enamel, and strengthen bones (prevent osteoporosis).

- Deficiency causes tooth decay and dental caries (cavities).

- Drugs that deplete fluoride: Calcium supplements and calcium- and aluminum-containing antacids reduce fluoride absorption (separate intake of fluoride from these by two hours).

- Supplements are available by prescription and are recommended only for children living in areas with low water fluoride concentrations; rarely required for adults. People who consume well water should have the fluoride content of their water tested.

\section{Iodine}

- Required to make thyroid hormones, which regulate metabolism, energy production, and body temperature, and are essential for growth and reproduction.

- Used for prevention of radiation-induced thyroid cancer in those with iodine deficiency and to treat fibrocystic breast disease. Deficiency may occur in those who do not consume salt, fish, or sea vegetables and is becoming more common in the general population due to restrictions on salt intake for blood pressure.

- Deficiency reduces thyroid hormone production, causing hypothyroidism, fatigue, weight gain, goiter, miscarriage, birth defects, and stunted growth. It is also the most common cause of brain damage worldwide.

- Drugs that deplete iodine: potassium iodide, possibly resulting in hypothyroidism.

\section{Micronutrients}

- Other interactions: Amiodarone (heart drug) contains high levels of iodine and may affect thyroid function; potassium iodide may decrease the anticoagulant effect of warfarin.

- A deficiency of selenium, vitamin A, or iron can worsen iodine deficiency. Foods containing goitrogens — such as cabbage, broccoli, cauliflower, Brussels sprouts, and soybeans-inhibit the synthesis of thyroid hormone. These foods are a concern only for those who are iodine deficient and consume high amounts of them. Cooking deactivates the goitrogens.

- Supplements are rarely necessary, but should be considered in pregnant and lactating women if dietary iodine is insufficient to meet the RDA. A daily prenatal supplement providing $150 \mathrm{mcg}$ of iodine will help to ensure that pregnant and breast-feeding women consume sufficient iodine during these critical periods. 
Iron

- Required to produce hemoglobin and myoglobin (proteins involved in the transport and storage of oxygen) and amino acids (carnitine); required for cellular energy production; produces enzymes that have antioxidant effects; supports DNA synthesis and immune function.

- Used for prevention of anemia in pregnancy and in others at risk, and in the treatment of restless legs syndrome.

- Deficiency is common, especially in women with heavy menstrual bleeding and during pregnancy (increased needs for baby), vegetarians, and those with malabsorption syndromes (celiac disease), bleeding ulcers, copper deficiency, and in surgery.

- Deficiency leads to depleted iron stores, impaired red blood cell formation, and anemia. Symptoms include fatigue, paleness, headache, hair loss, brittle nails, rapid heart rate, increased risk of infections, and rapid breathing on exertion.

- Drugs that deplete iron: antacids, cimetidine, ranitidine, omeprazole, lansoprazole, aspirin, anti-inflammatory drugs, and cholestyramine.

- Iron supplements can bind to and reduce absorption and efficacy of levodopa, levothyroxine, methyldopa, quinolones, tetracyclines, bisphosphonates, and zinc and calcium supplements. To avoid this, separate intake of iron supplements from these products by two hours.

- Vitamin C-rich foods and supplements enhance the absorption of nonheme iron (form of iron found primarily in plants).

- A multivitamin/mineral complex providing the RDA is recommended for most premenopausal and pregnant women and those at risk of deficiency.

- Men and post-menopausal women should choose iron-free multivitamin/mineral supplements to avoid iron excess.

\section{Manganese}

- Required for the production and activation of enzymes that are involved in energy metabolism; bone, cartilage, and collagen formation; and the production of antioxidants.

- Deficiency is uncommon, but may occur in those with epilepsy, hypoglycemia, diabetes, schizophrenia, and osteoporosis.

- Deficiency symptoms: impaired growth and reproductive function, skeletal abnormalities, impaired glucose tolerance, and altered carbohydrate and fat metabolism.

- Drugs that deplete: magnesium-containing antacids and laxatives and tetracycline.

- Absorption is reduced by calcium, phosphate, and iron.

- Supplements beyond the amount provided by diet and/or a multivitamin and mineral complex are not necessary. 


\section{Molybdenum}

- Required for the production of enzymes that are cofactors in amino acid metabolism, formation of uric acid, and the metabolism of drugs and toxins.

- Deficiency is extremely rare and may occur in those with a rare genetic condition; deficiency causes seizures, developmental delays in neonates, tachycardia, brain damage, and coma.

- Drugs that deplete: high intakes of copper or sulphate.

- Supplements beyond the amount provided by diet and/or a multivitamin and mineral complex are not necessary.

\section{Selenium}

- Component of enzymes that function as antioxidants; involved in detoxification; converts thyroid hormone to its active form; supports immune function; enhances the antioxidant activity of vitamin $\mathrm{E}$.

- Used to strengthen immune function and prevent infection, to protect against colon and prostate cancer, and to prevent oxidative stress and support immune system function in those with HIV/AIDS.

- Deficiency is uncommon, but may occur in those with poor diets, those who live in areas where the soil is depleted in selenium, Crohn's disease, and malabsorption syndromes (celiac disease).

- Symptoms of deficiency: muscular weakness and wasting, cardiomyopathy (inflammation of the heart), pancreatic damage, and impaired immune function.

- Drugs that deplete: valproic acid and corticosteroids (prednisone).

- Supplements beyond the amount provided by diet and/or a multivitamin and mineral complex may be necessary for some individuals.

\section{Zinc}

- Involved in numerous enzyme reactions; required for growth and development, immune and neurological function, reproduction and regulation of gene expression; stabilizes the structure of proteins and cell membranes.

- Used to support immune function, reduce severity and duration of the common cold, and delay the progression of macular degeneration.

- Severe deficiency is rare, except in those with a genetic disorder, severe malnutrition or malabsorption, severe burns, or chronic diarrhea. Marginal deficiencies are common in malnourished people, vegetarians, pregnant women, the elderly, and those with celiac disease, Crohn's disease, colitis, and sickle cell anemia.

- Symptoms of deficiency include impaired growth and development, skin rashes, severe diarrhea, immune system deficiencies, impaired wound healing, poor appetite, impaired taste sensation, night blindness, clouding of the corneas, and behavioural disturbances.

- Drugs that deplete: diuretics, anticonvulsants, iron supplements, penicillamine, ACE-inhibitor drugs, acid-reducing drugs, and oral contraceptives. 
Asian Journal of Applied Science and Technology Volume 4, Issue 3, Pages 215-284, July-September 2020

- Zinc supplements can reduce copper levels, so look for a multivitamin that contains copper as well as zinc.

- Zinc supplements can reduce absorption of antibiotics (tetracycline and quinolones), so separate intake of zinc supplements from these products by two hours.

- Since the average zinc intake is below the RDA and many conditions and drugs deplete zinc levels, a supplement should be considered. Most multivitamin and mineral complexes provide at least the RDA for zinc.

\section{Electrolytes}

\section{Potassium}

- Required to maintain fluid balance; required for nerve conduction and muscle function; cofactor for enzymes involved in energy production and carbohydrate metabolism.

- Used for prevention of stroke, osteoporosis, kidney stones, and in the treatment of high blood pressure.

- Deficiency (hypokalemia) is common and caused by prolonged diarrhea or vomiting, alcoholism, kidney failure, laxative abuse, anorexia, or magnesium deficiency. Deficiency symptoms include fatigue, muscle weakness and cramps, bloating, constipation, and abdominal pain. Severe hypokalemia may result in muscular paralysis or abnormal heart rhythms.

- Drugs that deplete: furosemide, hydrochlorothiazide, corticosteroids, pseudoephedrine, caffeine, and high-dose penicillin. Drugs that enhance potassium (may cause hyperkalemia): Spironolactone, triamterene, amiloride, ACE-inhibitors, anti-inflammatory drugs (ibuprofen), heaparin, digoxin, and beta-blockers.

- The average dietary potassium intake is about $2,300 \mathrm{mg} / \mathrm{day}$ for women and 3,100 $\mathrm{mg} /$ day for men. Evidence suggests that diets supplying at least 4,700 mg per day are associated with a decreased risk of stroke, hypertension, osteoporosis, and kidney stones, and this is the AI level set by the Institute of Medicine.

- Multivitamin/mineral complexes typically provide $99 \mathrm{mg}$ of potassium per serving. Depending on dietary intake and personal risk factors, additional potassium supplements may be necessary for some people.

- Take supplements with meals or choose a microencapsulated form to reduce the risk of upset stomach.

\section{Sodium}

- Regulates fluid balance along with potassium; required for nerve conduction and muscle function; assists absorption of chloride, amino acids, glucose, and water; regulates blood volume and blood pressure.

- Excess sodium intake is linked to gastric cancer, osteoporosis, high blood pressure, and kidney stones. Reducing sodium intake may help to reduce the risk of these conditions. Deficiency is rare; low blood levels of sodium (hyponatremia) may be caused by fluid retention or excess sodium loss (excessive sweating, prolonged exercise, severe and prolonged vomiting and diarrhea, and kidney disease).

- Symptoms of hyponatremia include headache, nausea, muscle cramps, fatigue, confusion, and fainting. Severe cases may lead to swelling of the brain, seizures, coma, and brain damage. 
Asian Journal of Applied Science and Technology

Volume 4, Issue 3, Pages 215-284, July-September 2020

- Drugs that deplete sodium: diuretics, anti-inflammatory drugs, carbamazepine, codeine, morphine, and some antidepressants.

- Supplements are rarely necessary, except in the above-mentioned conditions.

- The AI level for sodium and sodium chloride (salt) is based on the amount needed to replace losses through sweat in moderately active people and to achieve a diet that provides sufficient amounts of other essential nutrients. Most adults consume an amount much greater than the AI

\section{Nutrition Requirement in different Life Style Disorders-}

\section{Acne}

Acne is a chronic disorder of the skin's sebaceous glands (oil glands), leading to the development of comedones, also known as pimples, or more commonly "zits."

Almost every teenager will have an occasional acne outbreak, and approximately 40 percent of teens have severe cystic acne. Even those 30 and older are affected. Acne is not life threatening, but it can leave physical and emotional scars.

\section{Causes of Acne}

During puberty hormones called androgens trigger the sebaceous glands to grow and produce more sebum (oil). Irregular shedding of skin cells lining the hair follicle can lead to clumping and cause the pores to clog. A type of bacteria called Propionibacterium acnes, which normally lives in the skin, invades the clogged pore and begins to grow, creating inflammation and irritation. The result is a plugged, inflamed follicle that develops into a pimple.

\section{Causes of Acne}

- Allergies: Reactions to foods, medications, or environmental chemicals

- Cosmetics or exposure to airborne grease (working in a fast-food restaurant)

- Exposure to extreme temperatures

- Family history: Having a parent with acne

- Hormonal changes in teenagers, premenstrual women, and during pregnancy

- Medications: Use of hormones such as testosterone or cortisone

- Stress

\section{Dietary Recommendations}

\section{Foods to include}

- Fibre supports detoxification and elimination flaxseed is particularly helpful as it is high in both fibre and essential fatty acids.

- Yogurt contains beneficial bacteria. Seasonal fruits and vegetables are good to eat. 


\section{AJAST}

\section{Foods to avoid}

- Food allergies can trigger acne breakouts.

- Sugar reduces immune function and may increase bacterial growth.

\section{Lifestyle Suggestions}

- Drink eight to 10 glasses of water daily.

-Wash your face morning and evening with warm water and a gentle cleanser.

- Do not scrub hard or use abrasive cleaners as this can irritate acne.

- Sauna or steam help to clear pores and aid in detoxification.

- Resist the temptation to pick or squeeze, which can cause tissue damage, infection, and scars.

\section{Recommended PUGOS Nutrition}

To improve acne and overall skin health, consider the following:

- Nourish your body and skin with a healthy diet, including lots of fibre rich seasonal Fruits \& Vegetables, good fats, and water.

- Keep your skin clean and avoid picking blemishes.

- Minimize wearing cosmetics. Use only water-based and hypoallergenic skin care products.

- For severe cases, where there are multiple lesions or cysts, consult a dermatologist.

\section{Alzheimer's Disease}

- Alzheimer's disease is a chronic, degenerative disease of the brain that impairs thinking, memory, and cognitive function.

- It is the most common form of dementia.

- Alzheimer's is not a normal part of aging, but it is more common in people as they age.

- The disease causes damage and death to brain cells.

\section{Cause of disease}

- In a healthy brain, there are billions of neurons (nerve cells) that generate electrical and chemical signals, which help us think, remember, and feel. In those with Alzheimer's the neurons begin to die, affecting the normal signaling in the brain.

- Research has shown that Alzheimer's disease involves oxidative and inflammatory processes

- The use of natural supplements can delay the progression of the disease and improve symptoms and quality of life. 


\section{AJAST}

Asian Journal of Applied Science and Technology Volume 4, Issue 3, Pages 215-284, July-September 2020

\section{Symptoms of Alzheimer's Disease}

- Confusion and disorientation

- Depression

- Difficulty performing familiar tasks (cooking, tying your shoes)

- Increasing forgetfulness

- Age: It is most common in those over 65

- Environmental toxicity: Pollution and smoking generate excess free radicals.

- Gender: Women are at greater risk.

- Head injury: Some studies show that trauma to the head increases risk of Alzheimer's.

\section{Dietary Recommendations}

\section{Foods to include}

- Fish (wild, organic salmon, halibut, sole, and cod) /flaxseed oil provide good sources of omega fatty acids, a polyunsaturated fat that has been found to reduce Alzheimer's risk by reducing inflammation and protecting the nerve cell membranes.

- Olive oil is a monounsaturated fat that is also helpful.

- Foods such as whole grains, vegetable oils, nuts, seeds, and egg yolks are good.

Two prominent clinical studies have demonstrated that omega fatty acid supplement was associated with a 60 percent reduction in the risk of developing Alzheimer's disease (JAMA, 2002: 288; 2266-2268 and Archives of Neurology, 2003: 60; 940-946).

\section{Foods to avoid}

Avoid fast foods, deep-fried foods, and baked goods and margarine containing hydrogenated oils; minimize saturated fat (red meat and high-fat dairy).

\section{PUGOS healthy Lifestyle Suggestions}

- Maintain a healthy body weight. Being obese and its consequences of high blood pressure and cholesterol are risk factors for Alzheimer's disease. Exercising regularly helps with weight management, and also increases blood flow to the brain.

$>$ According to one study, regular exercises (walking 15 minutes three times per week) reduced the risk of Alzheimer's and dementia by 40 percent in individuals over age 65 .

$>$ Mental exercises: keeping your brain active, especially in later years, reduces the risk of Alzheimer's.

B-vitamins: Support neurotransmitter function. Vitamin B is involved in nerve transmission and may be deficient in those with Alzheimer's Disease. 
Asian Journal of Applied Science and Technology

Volume 4, Issue 3, Pages 215-284, July-September 2020

Fish oils/flaxseed supplement: Reduce inflammation and protect the nerve cell membranes. Regular consumption of reduces Alzheimer's risk and is important for brain function.

\section{PUGOS Nutritional Therapy}

To improve cognitive function and delay the progression of Alzheimer's, consider the following:

1. Eat more fish/flax seed oil, whole grains, vegetables, nuts, seeds, and olive oil.

2. Avoid saturated, trans fats, and aluminum-containing food ingredients like fast foods, deep-fried foods, and baked goods.

3. Get regular exercise such as walking.

4. Keep your brain active with games, puzzles, and exercises.

5. Consider supplements of liqimega and optigision gold

6. Synergistic therapy of liqimega and optigision gold BD dose will work best.

\section{Nutrition for Allergy/Allergic Rhintis/Sinusitis}

Approximately 20 percent of adults and children - suffer from allergies. These allergies occur when the immune system overreacts upon exposure to allergens, substances such as trees, grass, or flower pollen. Some people have persistent, year-long allergies that are triggered by environmental factors such as house dust, mould, animal dander, dust mites, and air pollution.

The immune system responds to these otherwise harmless substances as invaders, similar to how it would react to viruses or bacteria. It produces an antibody against the allergen called immunoglobulin $\mathrm{E}$ (IgE), which triggers the release of inflammatory chemicals - histamine, leukotrienes, and prostaglandins. Histamine is responsible for the notorious allergy symptoms of itchy, runny eyes and nose, and sneezing; leukotrienes cause excess mucus production; and prostaglandins trigger inflammation.

Our immune system is primed to respond when faced with a foreign invader.

- Cough

- Fatigue

- Headache

- Irritability

- Itchy eyes, nose, roof of mouth, or throat

- Sinus pressure and pain

- Sneezing

- Runny nose and congestion

- Watery eyes 
Asian Journal of Applied Science and Technology

Volume 4, Issue 3, Pages 215-284, July-September 2020

Having allergies increases your risk of developing other inflammatory diseases such as asthma and eczema. Prolonged sinus congestion can increase the risk of sinusitis (infection or inflammation of the sinuses).

\section{Dietary Recommendations}

\section{Foods to include}

- Fruits and vegetables provide antioxidants that support healthy immune function.

- Fish, Flax seeds, and nuts contain healthy fats that help reduce inflammation.

- Peppers, onions, and garlic help thin and reduce mucus.

- Wasabi (Japanese horseradish) clears the sinuses.

\section{Foods to avoid}

- Mucus-forming foods such as dairy products, refined flours, and saturated and hydrogenated fats can trigger inflammation and should be avoided.

\section{Lifestyle Suggestions}

- Know your triggers and try to avoid them-pollen production is highest between 5 a.m. and 10 a.m., so avoid the outdoors during this time.

- Heavy rain reduces pollen in the air, making post-shower outings safer.

- Wear a mask when cutting the grass or gardening.

- Don't dry your clothes outside, as they can collect pollen.

- Use an air purifier to remove allergens from your home.

- Keep your windows closed to prevent pollen from blowing into your home.

- Use a high-efficiency particulate air (HEPA) fi lter on your air conditioner and furnace and change it monthly.

- Use a vacuum with a double bag and a high-efficiency particulate air (HEPA) fi lter.

- Don't keep cut flowers in the house and minimize indoor plants. Wet dirt allows mould to grow.

\section{Recommended Pugos Nutrition}

Immunize/ Colostramin, Astashine, Liquimega,

\section{Antioxidant Nutrition}

Nutrition for Protection against Pollution, tobacco, cigarette smoke, drug addiction, illness, stress, alcohol, medications, trauma, cold, infections, poor diet, toxins, radiation.

Antioxidants are our first line of defense against free radical damage, and are critical for maintaining optimum health and wellbeing. The need for antioxidants becomes even more critical with increased exposure to free radicals. Pollution, cigarette smoke, drugs, illness, stress, and even exercise can increase free radical exposure. 
Because so many factors can contribute to oxidative stress, individual assessment of susceptibility becomes important. Antioxidants terminate the chain reactions by removing free radical intermediates, and inhibit other oxidation reactions. They do this by being oxidized themselves, so antioxidants are often called as reducing agents. Antioxidants are widely used as ingredients in dietary supplements and have been investigated for the prevention of diseases such as cancer, coronary heart disease and even altitude sickness.

Oxidative Stress occurs in response to excessive levels of cytotoxic oxidants and free radicals in the environment. Antioxidant is a chemical compound or substance that inhibits oxidation to protect body cells from the damaging effects of oxidation.

The term "oxidative stress" has been coined to represent a shift towards the pro-oxidants in the pro-oxidant/antioxidant balance that can occur as a result of an increase in oxidative metabolism. Increased oxidative stress at the cellular level can come about as a consequence of many factors, including exposure to alcohol, medications, trauma, cold, infections, poor diet, toxins, radiation, or strenuous physical activity. Protection against all of these processes is dependent upon the adequacy of various antioxidant substances that are derived either directly or indirectly from the diet. Consequently, an inadequate intake of antioxidant nutrients may compromise antioxidant potential, thus compounding overall oxidative stress.

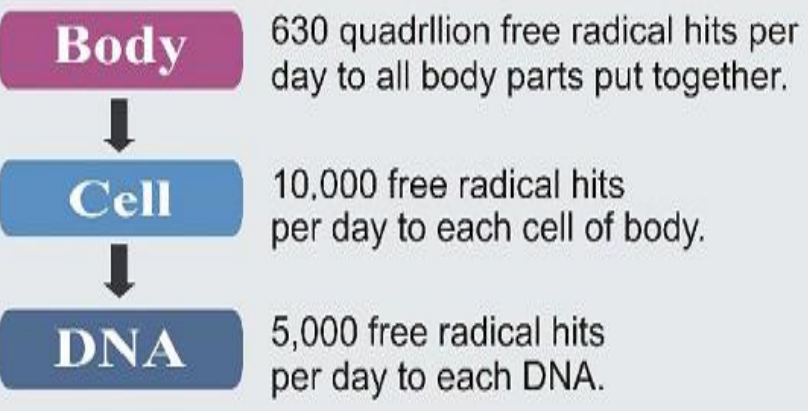

Oxidative Stress to Body, Cell \& DNA

\section{Oxidative Stress and Human Disease}

Oxidative damage to DNA, proteins, and other macromolecules has been implicated in the pathogenesis of a wide variety of diseases, most notably heart disease and cancer.

Clinical intervention trials suggest that antioxidants may play a pivotal role in preventing or slowing the progression of wide variety of diseases, such as heart disease and some forms of cancer.

\section{Conditions Associated with Oxidative Damage}

- Atherosclerosis

- Cancer

- Pulmonary dysfunction

- Cataracts 
- Arthritis and inflammatory diseases

- Diabetes

- Shock, trauma, and ischemia

- Renal disease and hemodialysis

- Multiple sclerosis

- Pancreatitis

- Inflammatory bowel disease and colitis

- Parkinson's disease

- Neonatal lipoprotein oxidation

- Drug reactions

- Skin lesion \& Aging

\section{What Are Antioxidants?}

Antioxidants are found in many foods. They work to keep our cells healthy by protecting them from damage by free radicals (molecules responsible for aging, tissue damage, and some disease). Free radicals damage cells in a process called oxidation. Oxidation results from everyday body functions such as breathing or walking, but certain processed and fatty foods, toxic substances, and sunlight can increase its effects. Antioxidants help repair damaged cells, which can prevent diseases, including cancer. A diet rich in a variety of plant-based foods provides all of the antioxidants the body needs. Research shows that vitamins, minerals, and phytochemicals from whole foods interact to boost their disease-fighting effects. These nutrients benefit both healthy people and those fighting disease. This is why it is important to focus on eating nutrient-rich foods rather than focusing on a single nutrient in supplement form.

\section{How Do Antioxidants Operate?}

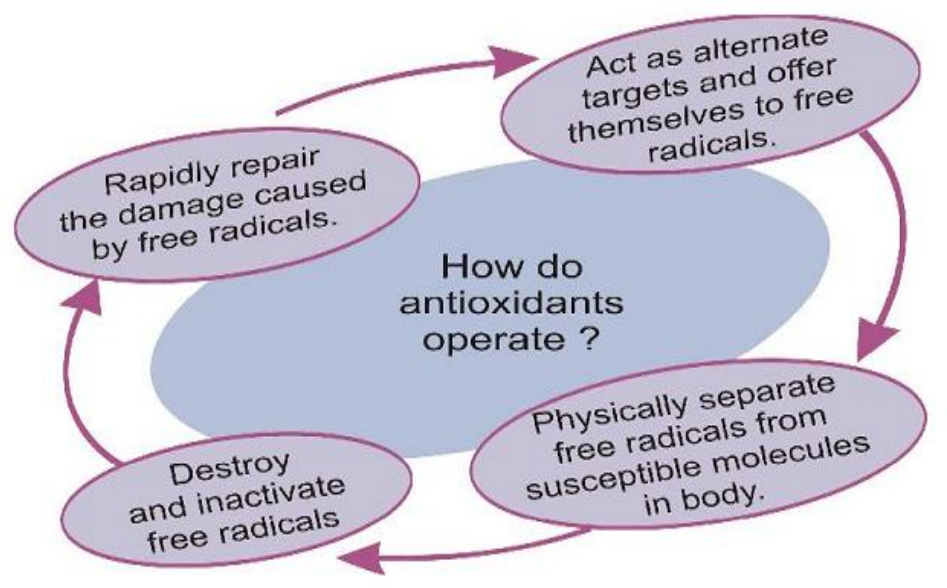




\section{Natural Antioxidants to Neutralize Free Radicals}

To protect the cells and organ systems of the body against reactive oxygen species, humans have evolved a highly sophisticated and complex antioxidant protection system. It involves a variety of components, both endogenous and exogenous in origin, that function interactively and synergistically to neutralize free radicals.

\section{Recommended Pugos Supplements}

ASTASHINE (World's Most powerful Antioxidant), NUTREASE

\section{Nutrition for Body Detoxification}

\section{Why body detoxification is important?}

- Due to pollution, tens of thousands of toxic chemicals have been introduced into our environment. Added to this, use of alcohol, tobacco, prescription and illicit drugs has made it a challenging task for the body to get rid of these chemicals. Removal of these toxic elements or body detoxification becomes very important for a healthy living.

- The Liver carries the greatest burden of detoxifying the foreign substances that are toxic to the body. It plays a major role in the detoxification of numerous substances in the body, whether these substances come from the environment, from food or formed within the body (from hormones) and other substances).

\section{What is Nudetox?}

- Nudetox is a blend of food ingredients, nourishing herbs, antioxidants, amino acids and minerals together are mainly required for liver detoxification. These beneficial ingredients help to support the intensive cleaning of the tissues. The unique combination promotes healthy detoxification.

\section{Pharmacological Action of Nudetox capsules}

\section{Grape seed Extract}

- Antioxidant with its flavonoid phytonutrients helps to detoxify liver from toxins, drugs and supports immunity.

\section{$N$-Acetyl Cysteine}

- It is a precursor of L-glutathione and eliminates xenobiotics and intestinal endotoxins.

\section{Alpho Lipoic Acid}

- Powerful antioxidant that helps to remove toxins and byproducts of fat metabolism.

\section{Silymarin Extract}

- A natural herb that has antioxidant and anti-inflammatory properties. It helps to protect the liver functions against environmental toxins.

\section{Taurine and Glycine}

- Helps to support healthy metabolism and elimination of xenobiotics. They help to support the metabolic integrity of mitochondria. 


\section{AJAST}

Asian Journal of Applied Science and Technology Volume 4, Issue 3, Pages 215-284, July-September 2020

\section{Minerals}

- To neutralize the toxins involved in protein metabolism.

\section{Amino acids}

- Helps to eliminate heavy metals such as mercury and arsenic and also helps to support glutathione production and healthy cell cycling activity. They help to manufacture phase I detoxification enzymes.

\section{Indications}

NUDETOX CAPSULES are indicated for Nutritional Detoxification \& Cleansing of body's toxins from internal organs and tissues.

\section{Contra-Indications}

Known contraindications to any ingredients of the supplement.

\section{Dosage and Directions for Use}

Take 1-2 Nudetox capsules daily for up to three months.

It is taken preferably with meals or as directed by a physician, licensed nutritionist or certified trainer.

\section{Safety}

Nudetox capsules have an excellent safety record in both animal \& human investigations, should be considered as a supplement of choice.

Nudetox capsules are generally regarded as safe when taken in the recommended doses; however, mild reactions can include gastrointestinal problems, such as nausea.

Nudetox capsules are generally well tolerated. Because of lack of long-term safety data, it should be avoided by pregnant women and nursing mothers.

\section{Side-Effects}

Epigastric pain/tenderness, heartburn, diarrhea and nausea, flushing.

\section{Special Precautions}

Take Nudetox capsules with or directly after meals to lessen the possibility of gastrointestinal upset.

It should be avoided by pregnant women and nursing mothers.

\section{Storage Conditions}

Store in a cool \& dry place, protected from light. Keep out of reach of children.

STORAGE LIFE IS 2 YEARS.

The preparation should not be used after the expiry date.

Recommended Nutrition for Detoxification is NUTREASE+ NUDETOX 14 day's course. 


\section{AJAST}

Asian Journal of Applied Science and Technology Volume 4, Issue 3, Pages 215-284, July-September 2020

\section{Nutrition for Cancer}

Cancer is one of the most feared diseases and rightfully so. It is the leading cause of premature Deaths.

Cancer is a disease that starts in our cells. Our bodies are made up of millions of cells grouped together to form tissues or organs, such as muscles, skin, bones, and organs. Cancer occurs when there is an abnormal growth of cells, which can form lumps or tumours, or can spread through the bloodstream and lymphatic system to other parts of the body. Tumours can be either benign (non-cancerous) or malignant (cancerous). Benign tumour cells stay in one place in the body and are not usually life-threatening.

Malignant tumour cells are able to spread to invade nearby tissues and other parts of the body, which is a process called metastasis. When a malignant tumour spreads, it often causes swelling of nearby lymph nodes. Finding cancer early and getting treatment before it spreads can greatly help improve your chances of survival.

\section{Cancer Causing Factors}

- Free radical damage,

- Genetics,

- diet, and lifestyle,

- Family history, only about 5-10 percent of cancers is attributed to faulty genes. Having a family history

\section{Signs or Symptoms of Cancer}

- A lump or area of swelling under the skin or bloating in pelvic area

- A mole that is asymmetrical, has an irregular border, is dark in colour, or grows in size or thickness

- Change in bowel or bladder habits

- Difficulty swallowing, upset stomach, nausea, or vomiting

- Persistent cough, hoarseness, or a cough that brings up blood

- Persistent low-grade fever

- Rectal bleeding or blood in the urine or stool

- Recurrent infections or wounds that do not heal

- Unexplained pain, especially in the bones, breasts, or pelvic area

- Unexplained vaginal discharge or bleeding

- Unexplained weight loss and loss of appetite

- Unusual fatigue, headaches

- Becoming sexually active at a young age or having multiple partners increases the risk of cervical cancer. 
- Exposure to environmental toxins such as tobacco, pollution, asbestos, heavy metals (arsenic, lead, nickel), and radiation (X-rays), and handling of petroleum products.

Smoking is responsible for 30 percent of all cancer deaths.

Chlorinated drinking water increases the risk of bladder cancer.

Some substances used in pesticides are classified as known, probable, or possible carcinogens.

- Excessive sun exposure or sunburn during childhood increases the risk of skin cancer.

- Genetics: Family history of cancer increases risk.

- Heavy alcohol consumption increases risk.

- High insulin levels (hyperinsulinemia) and insulin resistance increase risk.

- High intake of saturated fat, trans fats, and high-glycemic foods (refined starches and sugar); low intake of fibre; consumption of pesticides, food additives; nutritional deficiencies (lack of antioxidants) increase risk.

- Lack of exercise increases your risk of several types of cancer.

- Obesity increases risk of breast, endometrial, cervical, ovarian, and gallbladder cancer for women and colon and prostate cancer for men.

- Poor liver function can increase risk. The liver plays a key role in removing toxins.

- Stress hampers immune function and may increase the risk of cancer (particularly breast cancer). Stress may also worsen recovery in those with cancer.

- Use of the hormones estrogen and progestin (HRT) increase the risk of breast cancer; risk is greatest with higher dosages for long periods of time (longer than five years).

- Weakened immune function, exposure to viruses and fungal infections increase risk.

\section{Treatment}

The treatment of cancer depends on the location, stage of cancer, existing health status, and many other factors. Today there are numerous options for medical treatment including chemotherapy, radiation, surgery, and hormone therapy. There are a variety of important screening tests that can help in the early detection of cancer, such as:

- Breast self-exams, mammograms, and thermography scans

- Digital rectal exams

- Pap test (for cervical cancer) and pelvic exam

- Prostate exam and PSA test

- Skin exam

- Testicular exams 
Asian Journal of Applied Science and Technology

Volume 4, Issue 3, Pages 215-284, July-September 2020

Numerous studies have shown that good nutrition and various lifestyle measures can significantly reduce the risk of cancer. For those who have cancer, a good nutritional program can help prevent weight loss, aid digestion, support immune function, and boost energy levels. Supplements can also play an important supportive role.

\section{Dietary Recommendations}

\section{Foods to include}

- Boost fibre intake: aim for 25-30 g daily of soluble and insoluble fibre. Eat 2 tbsps., of milled flaxseed every day: it provides fibre and contains compounds that help in the prevention of cancer. Recent research found that it can also slow the growth of prostate cancer.

- Choose healthy fats such as olive oil and flaxseed oil.

- Drink green tea, which is a potent antioxidant, and studies have linked drinking green tea to a lower risk of several cancers.

- Drink lots of purified water.

- Eat cancer-fighting foods such as broccoli, cabbage, cauliflower, Brussels sprouts, kale, tomatoes, garlic, onions, and carrots.

- Eat cancer-fighting herbs and spices like ginger, cayenne, rosemary, oregano, and curcumin.

- Eat lots of vegetables and fruit (10 servings daily). Choose organic produce as much as possible to avoid ingestion of pesticides.

- Eat small, frequent meals, which are easier on digestion and also help improve blood sugar control.

- Fermented soy products (tofu, tempeh and miso) appear to have cancer-fighting properties.

- Ginger tea may be helpful for managing nausea caused by chemotherapy.

- Yogurt and fermented dairy can aid digestion and intestinal function.

Consider doing a detoxification program as best preventive therapy for cancer.

\section{Eat Plant Based Diet}

Overwhelming evidence from numerous clinical trials indicates that a plant-based diet can reduce the risk of cancer. In 1992, a review of 200 studies showed that cancer risk in people consuming diets high in fruits and vegetables was only one-half that in those consuming few of these foods. It is clear that there are components in a plant-based diet that can reduce cancer risk.

- (Nutrition and Cancer, 1992; 18 (1): 1-29). Cancer 155

\section{Foods to avoid}

- Reduce intake of saturated fat to less than 10 percent of total calories by limiting animal and dairy products. Avoid trans fats (found in many processed/snack foods and fried foods) completely. 
- Avoid preservatives (nitrates and sulphites) and chemical food additives.

- Eating charred meat has been associated with increased risk of breast cancer

- Minimize alcohol, as it is hard on liver and immune function. Heavy drinking increases the risk of cancers of the mouth, esophagus, breast, colorectal, and stomach. If you drink, limit yourself to one or two drinks daily and choose red wine as it contains antioxidants that may offer cancer-protecting properties.

- Minimize eating sugar and refined starches, which quickly raise blood sugar and insulin levels, increasing the risk of insulin resistance, which is associated with increased risk of certain cancers.

\section{Lifestyle Suggestions}

- Get regular exercise. Aim for one hour of moderate-intensity activity each day, such as walking, cycling, or swimming.

- Don't smoke, and avoid second-hand smoke and highly polluted areas.

- Manage your stress. Try yoga, meditation, and breathing techniques.

- Drink purified water. Several long-term studies have found that drinking chlorinated tap water increases the risk of cancer, particularly bladder cancer.

- Avoid storing food in soft plastic containers. Use glass or hard plastic (which contains high density polyethylene).

- Use stainless steel cookware. All non-stick cookware is made up of a chemical called PTFE (polytetrafl uoroethylene). When heated to high temperatures (greater than $572^{\circ} \mathrm{F}$ ), they can create fumes that contain a suspected cancer-causing chemical, tetrafluoroethene-TFE. C

\section{Top Recommended Supplements}

Ginseng: Studies have shown benefits for reducing fatigue, nausea and vomiting, and improving well-being in those undergoing cancer treatment. American ginseng has been shown to reduce fatigue in those with breast, lung, colon, and other forms of cancer. It may also increase the effectiveness of treatment for breast cancer. Panax ginseng has been shown to improve energy and well-being in those with cancer. Regular use of Korean ginseng was found to lower overall cancer risk.

IP6 (inositol hexaphosphate): A supplement derived from rice bran. Animal research and preliminary human studies have shown that it has significant anti-cancer effects. It can help in the prevention of cancer and has growth-regulating effects on various cells and tissues, including those of the colon, breast, and prostate. Dosage: 4-8 g daily.

Multivitamin/mineral complex: Ensures that your body gets essential nutrients to support immune function and overall health. Look for a product that provides a full range of nutrients and is free of dyes and artificial chemicals. Dosage: Take daily. Consider extra antioxidants as it is difficult to obtain therapeutic dosages from a multivitamin. Dosages: 200-400 mcg selenium, 400-800 IU vitamin E (natural mixed tocopherols), and 500-2,000 mg vitamin C. Antioxidants may offer benefits for those undergoing chemotherapy. 


\section{Ginseng and Cancer}

According to research at Vanderbilt University in Nashville, TN, "Ginseng use after cancer diagnosis, particularly current use, was positively associated with quality of life scores, with the strongest effect in the psychological and social well-being domains.

Additionally, quality of life improved as cumulative ginseng use increased" (American Journal of Epidemiology, 2006: 163 (7); 645-653).

Probiotics: Replenish friendly bacteria that are depleted by treatment with chemotherapy and antibiotics. Probiotics aid digestion, support immune function and detoxification, and assist in nutrient absorption. They may also help to minimize diarrhea and upset stomach caused by chemotherapy and radiation. Dosage: One billion live cells twice daily.

\section{Recommended Pugos Supplements}

\section{CURCUMET, ASTASHINE, NUTREASE}

Regular Detoxification of Body with Nudetox+Nutrease helps to prevent cancer. Nutrease is helpful in cancer patients as it is natural plant based diet.

\section{Nutrition for Cataract}

Cataracts are cloudy spots that develop on the normally clear lens of the eye. Initially they might not be noticeable; however, as they progress, cataracts cause gradual clouding and loss of vision.

Cataracts are the leading cause of blindness. They are most common among older adults over 65 years have visual impairment due to cataracts. This can affect normal daily activities, making it difficult to read, drive a car, and walk safely. In most people cataracts occur due to age-related changes in the lens. The lens is comprised mainly of protein fibres and water. With age, the lens undergoes changes; it becomes thicker, less flexible, and less transparent. The protein fibres break down and begin to clump together, creating cloudy spots on the lens, which become progressively worse.

Cataracts tend to develop slowly in both eyes at the same time unless they are caused by an injury that affects only one eye. Cataracts that become completely white, known as overripe (hypermature) cataracts, can cause inflammation, pain, and headache.

Cataracts are not considered a "normal" part of aging, yet they do primarily affect older adults, likely due to lifetime exposure to free radicals from smoking, chemicals, UV light, and other factors that damage the lens.

\section{Dietary Recommendations}

\section{Foods to include}

- Research shows that a diet high in fruits and vegetables can help prevent cataract development because these foods provide excellent sources of antioxidants, which help reduce free radical damage. 
- Foods high in antioxidants include berries (acai, blueberry, and cranberry), cherries, tomatoes, peppers, carrots, grapes, mangos, and citrus fruits.

- Kale, collard greens, spinach, and broccoli are great sources of the carotenoids lutein and zeaxanthin, which lower the risk of cataracts and macular degeneration.

\section{Foods to avoid}

- Fast food and processed foods contain hydrogenated (trans fats) and saturated fats, which generate free radicals.

- Limit alcohol, as it impairs the liver's ability to detoxify harmful chemicals from the diet and environment.

\section{Lifestyle Suggestions}

- Get regular eye exams at least every other year. While this won't prevent cataracts, early detection gives you a chance to take steps to slow or prevent their development.

- Don't smoke, as smoking is a known risk factor because it generates damaging free radicals. Avoid smoky environments and people who smoke, as second-hand exposure can be just as damaging.

- Wear proper-fitting sunglasses with UV protection and wide-brim hats to shield your eyes from the sun's damaging rays.

- Maintain a healthy body weight. Obesity is associated with increased risk of cataracts.

\section{How to Avoid Cataracts}

The majority of the research evaluating the effect of antioxidant supplements has found protective benefits with long-term use lasting five to 10 years. Cataracts develop slowly over time. Consistent and long-term use of antioxidants, along with lifestyle changes, is your best defence. (Archives of Ophthalmology, 2000: 118; 1556-1563).

\section{Pugos Recommended Nutrition}

\section{ASTASHINE}

\section{Nutrition for Constipation}

Constipation is a disorder of the lower gastrointestinal tract marked by a decrease in the frequency of stools, difficulty defecating, and/or the passage of dry, hard stools.

Most people experience an occasional change in bowel habits; when it is persistent, it is referred to as chronic constipation.

During the digestive process, food passes from the stomach to the intestine where nutrients and water are absorbed into the body. The waste products of digestion create a stool, which travels through the intestines with muscle contractions. It normally takes six to 24 hours to pass a stool. Anything that slows the passage of stools through the intestines or increases the amount of water absorbed by the body such as a lack of fibre, fluids, or physical activity; medication; or ignoring the urge to defecate can lead to constipation. 
Asian Journal of Applied Science and Technology

Volume 4, Issue 3, Pages 215-284, July-September 2020

Chronic constipation affects 31 percent of people between 19 and 65 years, and approximately 45 percent of people over 65 years. This can be a debilitating and uncomfortable problem, but there are a number of lifestyle recommendations that can help.

- Abdominal pain, bloating, and gas

- Bowel movements less than three times a week

- Passing hard stools

- Straining during a bowel movement

Chronic constipation can also cause bad breath, headache, fatigue, hemorrhoids (due to straining), and worsen varicose veins.

Severe constipation may cause fecal impaction, a mass of hardened stool.

- Age (more common in older adults)

- Diet (lack of fibre and/or fluids)

- Health conditions (irritable bowel, celiac disease, colon cancer, liver disease, and low thyroid)

- Lack of activity (sedentary or bedridden)

- Medications (sedatives, narcotics [codeine], chemotherapy, blood pressure drugs,

Parkinson's drugs, and iron supplements)

- Pregnancy (causes hormonal changes)

- Stress (reduces intestinal motility)

\section{Sherry's Natural Prescription}

\section{Caution: Do Not Overuse Laxatives}

Overuse of laxatives may cause dependence (lazy bowel syndrome), poor absorption of vitamins and nutrients (particularly potassium), dehydration, damage to the intestinal tract, and worsened constipation. Those who take laxatives for a long time may need to go off them slowly to allow the bowels to return to normal function.

\section{Dietary Recommendations}

\section{Foods to include}

- High-fibre foods improve bowel regularity. Eat whole-grain breads and cereals (made with wheat bran, whole oats, rye, and flaxseed), fresh fruits (especially strawberries, apples, and rhubarb), dried fruits, vegetables, and legumes. Broccoli, spinach, and kale contain magnesium, which improves gut motility.

- Aim for 25-35 g of fibre daily.

- Drink plenty of fluids, water is best, but pure fruit and vegetable juices and herbal teas are also fine. 


\section{Foods to avoid}

- Refined and processed foods are high in sugar and contain little fibre.

- Mucus-forming foods (dairy) slow the transit time of waste and can be constipating.

- Caffeine and alcohol are dehydrating and should be minimized.

\section{Lifestyle Suggestions}

- Increase physical activity, as exercise helps stimulate intestinal and bowel contractions. Try walking, biking, or swimming.

- Set aside time to have a bowel movement, such as after breakfast.

- Don't resist the urge. The longer you delay going to the toilet once you feel the urge, the more water that is absorbed from the stool and the harder it becomes.

- Acupuncture, massage, and reflexology may help by improving intestinal motility.

- Detoxification, a short-term cleanse or juice fast along with fibre supplements (psyllium, flaxseed), allows the body to focus on removal of waste. This is particularly helpful for chronic constipation.

\section{Recommended Pugos Nutrition}

Nutrease, Nudetox, Liquimega

\section{Nutrition for Diabetes}

Diabetes is a chronic disease where the body does not make enough insulin, or becomes insensitive (resistant) to the insulin that is produced.

When we consume food, it is broken down into glucose, which causes a rise in blood glucose levels. Insulin is a hormone secreted by the pancreas in response to that rise in blood sugar. Insulin's role is to transport glucose from the bloodstream into the cells to be used for energy.

There are three main types of diabetes.

Type 1 diabetes is responsible for approximately 10 percent of cases and occurs when the pancreas produces little or no insulin. The exact cause of type 1 diabetes is unknown, but it is thought that the immune system attacks and destroys the insulin-producing cells of the pancreas. Genetics may also play a role. Type 1 diabetes was previously known as juvenile diabetes or insulin dependent diabetes because it typically appears during childhood or adolescence, and people who get this form require insulin injections to manage their blood sugar.

Type 2 diabetes is the most common form, accounting for about 90 percent of people with diabetes. This form occurs when the pancreas does not produce enough insulin or when your cells become resistant to the action of insulin. Obesity, inactivity, and poor diet (eating too many high-glycemic foods) are some of the causes of type 2 diabetes. In the past, type 2 diabetes affected primarily adults. However, a growing number of children and adolescents are being diagnosed today. 
The third type of diabetes is gestational diabetes, a temporary condition that occurs during pregnancy. It affects approximately 3.5 percent of all pregnancies and involves an increased risk of developing diabetes for both mother and child.

All forms of diabetes can have serious consequences if left untreated. There is no cure for diabetes, but there is much that can be done from a lifestyle perspective to improve blood sugar control and prevent potentially life-threatening complications.

- Blurred vision: High blood sugar causes fluid to be pulled from all tissues, including the lenses of the eyes, which can affect vision.

- Fatigue and irritability

- Hunger: Your muscles and organs become energy depleted because insulin is not able to move glucose into your cells, which can trigger persistent hunger.

- Impaired wound healing or frequent infections.

- Increased thirst and frequent urination; as excess sugar builds up in your bloodstream, fluid is pulled from our tissues, which can make you thirsty, so we may drink and urinate more than usual.

- Weight loss: Even though food intake may be increased, weight loss can occur because your muscles and fat stores may shrink because they are not getting the necessary glucose.

\section{Dietary Recommendations}

\section{Foods to include}

- Cinnamon contains compounds that work synergistically with insulin, helping to reduce blood sugar levels. One study found benefits with just $1 / 2$ tsp daily.

- Add cinnamon to your cereal, oatmeal, or breakfast shakes.

- Chromium is essential for blood glucose regulation. It is found in brewer's yeast, whole grains (especially wheat germ), onions, and garlic.

- For a natural and healthy sugar substitute, try stevia or xylitol.

- High-fibre, low-glycemic (slow-release) carbohydrates such as whole grains (whole wheat, oats, brown rice, spelt), vegetables, fruits, and legumes help to balance blood sugar.

- Protein (lean poultry, meat, and fish) and healthy fats (nuts, seeds, olive oil, and flaxseed oil) in each meal will slow carbohydrate digestion and promote better blood sugar control.

To promote steady blood sugar levels, eat small, frequent meals (every three hours).

\section{Foods to avoid}

- Alcohol can cause either high or low blood sugar depending on how much you drink and if you are eating while drinking. Limit alcohol intake to no more than two drinks daily. 
- High-glycemic (quick-release) carbohydrates such as white bread and baked goods, refined cereals, potatoes, white rice, and sugar (candy, cookies, soda) cause rapid and profound increases in blood sugar, creating a problem for diabetics. Studies have also found that those who eat high-glycemic diets are also at increased risk of developing type 2 diabetes.

- Saturated fat (animal products such as meat and dairy) can worsen blood glucose control.

\section{Lifestyle Suggestions}

- Lose excess weight. Being overweight can impair insulin sensitivity.

- Get regular physical activity. Aim for 30 minutes to one hour of moderate intensity activity each day, such as brisk walking, cycling, or swimming. Exercise helps with weight management and also improves blood glucose control and insulin sensitivity.

- Don't smoke. People with diabetes who smoke are at greater risk for heart, kidney and eye disease, and nerve damage.

- Manage your stress. Stress triggers the release of hormones that impair insulin sensitivity. Try yoga, meditation, and other relaxation techniques.

- Practice good oral hygiene. Brush your teeth at least twice a day and floss daily to reduce the risk of gum infection.

- Take care of your feet. Diabetics are prone to nerve damage, which can make sores on the feet unnoticeable and delay wound healing. Inspect your feet daily for blisters or cuts. Apply moisturizer particularly to your heels.

- Have regular physicals and eye exams to screen for potential complications.

\section{Top Recommended Supplements}

Antioxidants: A powerful antioxidant that can help improve insulin sensitivity and reduce the risk of diabetic complications such as neuropathy and nephropathy (kidney disease). Dosage: 600-1,200 mg daily.

Chromium: An essential trace mineral that plays a role in sugar metabolism. It helps improve insulin sensitivity and glucose tolerance. Some studies have found that diabetics are deficient in chromium, and that supplements can help improve blood sugar management. chromium picolinate as this is the most widely studied form of chromium. Dosage: $400-1,000 \mathrm{mcg}$ daily.

Fibre: Helps improve blood glucose control and weight management. Studies involving fibre supplements of psyllium, oat bran, and glucomannan have shown benefits for diabetics.

B-vitamins: Essential for proper nerve function and energy metabolism. Take a B-complex or a multivitamin that contains at least $50 \mathrm{mg}$ of the B-vitamins.

Fenugreek: Seeds and supplements containing this herb have been shown to lower blood sugar and improve insulin sensitivity. Dosage: $15 \mathrm{~g}$ of powdered seeds with a meal or 1 or $2 \mathrm{~g}$ of an extract daily. 
Omega fatty Acids: Helps improve glucose tolerance, reduce triglycerides and cholesterol levels, and may help improve diabetic complications (neuropathy and nephropathy). Dosage: $400 \mathrm{mg}$ EPA and $200 \mathrm{mg}$ DHA daily.

Gymnema: Preliminary research shows that this herb can help stimulate insulin secretion and improve blood glucose control in those with both Type 1 and Type 2 diabetes. Dosage: $400 \mathrm{mg}$ once or twice daily of a product standardized to 25 percent gymnemic acid.

Magnesium: Required for energy metabolism and nerve function. People with diabetes tend to have low magnesium levels and a deficiency is associated with insulin resistance. Supplements can help improve insulin sensitivity and glucose control. Dosage: 200-600 mg daily.

Vitamin E: Helps to improve glucose tolerance and reduce glycosylation (binding of sugar to proteins in blood vessels). Many studies have found that it can prevent and reverse nerve damage and help protect against retinopathy and nephropathy. Dosage: 800 IU daily.

\section{Recommended Pugos Supplements}

Astashine/Astashine silver, liquimega, Optigision gold,

\section{Nutrition for Erectile Dysfunction (Impotence)}

Erectile dysfunction (ED), also known as impotence, is the inability to achieve or maintain an erection that is adequate for sexual performance. This is the most common sexual problem among men-almost every man will experience an episode of ED at one point in his life. Persistent ED affects approximately 25 percent of men over age 50 and this figure rises with age.

\section{Factors Effecting ED}

- Diabetes: Elevated blood sugar damages blood vessels in the penis. Diseases of the nerves, blood vessels, heart, prostate, and kidneys. Hormonal imbalances: Low testosterone, growth hormone, or DHEA levels

- Surgery or trauma that causes nerve damage (spinal cord injury, prostate surgery)

- Drugs: Antidepressants, beta-blockers, antihistamines, tranquilizers, and anti-cancer drugs

- Alcohol, marijuana, cocaine, and other illicit drugs. Smoking causes damage to blood vessels and nerves in the penis, affecting erectile function. Psychological factors: Stress, anxiety, depression, and lack of sleep.

\section{Diabetes and ED}

Diabetes is the leading cause of erectile dysfunction. Approximately 80 percent of men with diabetes develop erectile dysfunction, and it occurs at an earlier age.

\section{Dietary Recommendations}

\section{Foods to include}

- Fibre-rich foods help promote blood sugar balance. Zinc helps support testosterone production; the best food sources are pumpkin seeds, soybeans, oysters, and wheat germ. 
Asian Journal of Applied Science and Technology Volume 4, Issue 3, Pages 215-284, July-September 2020

- Vitamin E dilates blood vessels and improves blood flow; the best food sources include nuts and seeds, whole grains, and leafy green vegetables.

\section{Foods to avoid}

- Foods high in saturated and trans fats can be damaging to the blood vessels and reduce circulation (red meat, dairy, processed and fast foods).

- Minimize refined flour and sugar as these foods cause blood sugar fluctuations, which can be damaging to the blood vessels.

- Avoid alcohol.

\section{Lifestyle Suggestions}

- Exercise: Regular activity is essential to improve blood flow and circulation, reduce stress, and increase energy levels. Activities such as walking, squats, and lunges are particularly good for increasing blood flow to the pelvic area.

- Don't smoke. Smoking has serious consequences, as it damages blood vessels and nerves.

- Maintain a healthy body weight. Obesity leads to diabetes and heart disease, both of which can impact sexual function. Get adequate sleep at night and be aware that a lack of sleep can affect performance.

- Reduce stress whether through exercise, breathing techniques, or visualization. Find what works to promote relaxation and practice it regularly.

\section{Recommended Supplements}

Arginine: An amino acid used to make nitrous oxide, a substance that dilates blood vessels and increases blood flow. Dosage: $1,000 \mathrm{mg}$ three to five times daily.

Korean ginseng: Also called panax ginseng, Korean ginseng increases energy and the ability to maintain an erection; several studies have found it effective for improving erectile function.

Dosage: $1,800-2,700 \mathrm{mg}$ daily.

Antioxidants: Vitamins $\mathrm{C}$ and $\mathrm{E}$ and flavonoids (grape seed and pine bark) help improve blood flow and reduce oxidative damage to the blood vessels.

Ginkgo biloba: Opens blood vessels and increases blood flow to the penis, is particularly helpful for ED caused by antidepressant drugs or vascular problems (diabetics). Dosage: 120-200 mg daily.

Maca: Rich in the amino acid Arginine, which increases blood flow to the penis and histidine, which helps support orgasm and ejaculation; preliminary research has found it helpful. Dosage: 500-1,000 mg three times daily.

\section{Recommended Pugos Supplements}

ASTASHINE, M-RUSH Capsules 


\section{Nutrition to Eradicate Fibroids (Uterine)}

Uterine fibroids are non-cancerous tumours or growths of the uterus. They are also known as myomas, fi bromyomas, or leiomyomas. Despite their name, fibroids are not fibrous; they are comprised of connective tissue and muscle. They can appear in groups and grow either inside or outside of the uterus. Approximately 50 percent of women have fibroids; however, many are unaware. They can be smaller than a pea, or grow as large as a football. In rare cases fibroids can develop into cancer; however, most often they are not harmful, but may cause pain and discomfort when they become large and put pressure on the abdomen and bladder. Fibroids typically appear during the child-bearing years, particularly between the ages of 30 and 40 years, and then shrink in menopause. While the actual cause of fibroids is uncertain, it is known that their growth is stimulated by estrogen. Fibroids are becoming increasingly common today, which has been attributed to high amounts of xenoestrogens (estrogen-like compounds) in our food supply and environment.

\section{Environmental Estrogens}

Xenoestrogens are substances that have estrogen-like activity in the body. They are present in foods (fish, meat, and dairy) and in the environment (plastics, pesticides, and skin care products). Research suggests that xenoestrogens may play a role in uterine fibroids, certain cancers, and premature sexual maturity in young girls. Many women do not realize they have fibroids until it is discovered as a result of a pelvic examination or prenatal ultrasound. For those who develop large fibroid tumours, Signs and symptoms vary depending on location, and may include:

- Anemia (due to heavy bleeding)

- Backache or leg pains

- Constipation

- Heavy menstrual bleeding and prolonged periods

- Infertility and miscarriages

- Pelvic pressure or pain

- Urinary frequency or incontinence

- Age: Most common between the ages of 30 and 40 years

- Estrogen excess (promotes growth of fibroids)

- Family history (fibroids contain genetic alterations)

- Insulin-like growth factor (may stimulate fibroid growth)

- Race (fibroids are more common among Black women)

\section{Sherry's Natural Prescription}

Medications are also used to reduce estrogen levels and shrink fibroids; however, there are many side effects with these drugs. Progesterone cream is popularly used for fibroid treatment; however, in some women it can cause 


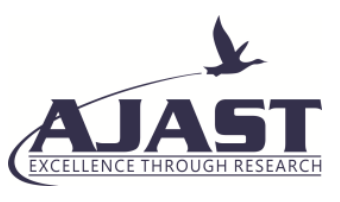

Asian Journal of Applied Science and Technology Volume 4, Issue 3, Pages 215-284, July-September 2020

fibroids to grow, so it should be used only under a physician's supervision. A holistic approach to treating fibroids is aimed at reducing levels of circulating estrogens and supporting liver function (the liver detoxifies estrogen). This can be achieved through dietary, lifestyle, and supplemental approaches.

\section{Dietary Recommendations}

\section{Foods to include}

- Fibre-rich foods such as fruits, vegetables, and whole grains will help extract excessive estrogen stores from the body.

- Cruciferous vegetables such as broccoli, cauliflower, and Brussels sprouts are particularly helpful, as they contain compounds that help the liver detoxify estrogen.

- Choose organic foods whenever possible to reduce ingestion of xenoestrogens from pesticides.

- Foods containing phytoestrogens (plant-based estrogens), such as soy foods and flaxseed, help balance estrogen levels.

- Artichokes, beets, carrots, dandelion greens, garlic, and onions contain compounds that support liver function and detoxification of hormones.

- Antioxidants block the negative effects of estrogen and help protect against estrogen-related cancers.

\section{Foods to avoid}

- Meat, dairy, and fish (farmed salmon) may contain dioxins and other xenoestrogens that can stimulate fibroid growth and worsen symptoms.

- Minimize sugar and caffeine, which can trigger pain and inflammation.

\section{Lifestyle Suggestions}

- Reduce stress, as it can cause hormonal changes that may worsen fibroid symptoms. Try yoga, , and breathing techniques.

- Regular exercise of moderate-intensity activity for one hour daily reduces stress and will help to maintain a healthy body weight.

- Apply castor oil to your pelvic area, cover with plastic and a hot towel. Leave in place for one hour and do this three times per week.

\section{Recommended Pugos Nutrition Supplements}

Nutrease, Optigision gold, Astashine, Curcumet

\section{Nutrition to Remove Gallstones}

Gallstones are solid deposits of cholesterol or mineral salts that form in your gallbladder or nearby bile ducts. It is estimated that about 20 percent of population have gallstones by age 60. In many cases they do not cause a problem 
Asian Journal of Applied Science and Technology Volume 4, Issue 3, Pages 215-284, July-September 2020

or symptoms. However, some people with gallstones develop a gallbladder attack, which causes abdominal pain, bloating, and nausea. The gallbladder is a pouch-shaped digestive organ that is located near the liver and is responsible for storing and concentrating bile, a greenish-brown fluid composed of bile salts, fatty compounds, cholesterol, and other chemicals produced by the liver.

\section{Dietary Recommendations}

\section{Foods to include}

- Beets, artichokes, and dandelion greens help to improve the flow of bile.

- Coffee is actually protective. Caffeine increases bile flow and stimulates gallbladder contractions.

In one study of 46,000 men, those drinking 2 or 3 cups of regular coffee per day had a 40 percent lower risk of gallstones compared with men who did not drink coffee.

- Eat small, frequent meals.

- Diets high in fibre are associated with a lower risk of gallstones. Eat more wheat and oat bran, milled flaxseed, legumes, vegetables, and fruits.

- Include lean protein (fish and poultry) and healthy fats (olive and flaxseed oil).

\section{Foods to avoid}

- Diets high in sugar are linked to increased risk of gallstones. Minimize high-sugar foods such as candy, soft drinks, pastries, and other sweets.

- Eating lots of saturated fat and cholesterol increases the risk of gallstones. Minimize red meat, high-fat dairy, eggs, deep-fried and processed foods.

\section{Lifestyle Suggestions}

- Maintain a healthy body weight. If you are overweight, follow a healthy diet (not fad diet) and boost activity levels to promote slow, gradual weight loss. Rapid weight loss can actually increase your risk of gallstones.

- Get regular physical activity every day.

\section{Recommended Pugos Supplements}

Astashine, Nutrease, liquimega

\section{Nutrition for Hirsutism}

Hirsutism is excessive hair growth in certain areas of a woman's face and body, such as the mustache and beard area, that creates a "male pattern" of hair. Women normally can have fine, pale, faintly visible hair in these areas, but heavy hair growth in a male pattern with coarse or colored hair is not expected.

Hirsutism in women means that hair follicles are being over-stimulated by testosterone or other androgen hormones. Androgens are the dominant sex hormones in men. Women normally have low levels of androgens. 
Hirsutism can be caused by abnormally high levels of androgens or abnormal stimulation of hair follicles even when androgen levels are normal.

In some cases, the extra androgen comes from medications such as certain progestins used in some brands of birth control pills or bodybuilding steroids that contain androgens or have some effects similar to androgens. Other medicines may indirectly cause the body to manufacture extra androgen hormones. These include some medicines to treat nausea, schizophrenia, agitation, epileptic seizures, migraine headaches, bipolar disorder, aggression and high blood pressure, as well as estrogens and opiate medications.

\section{Nutritional tips may help women stay at a good weight, which may help lower androgens in the body:}

Eat antioxidant foods, including fruits (such as blueberries, cherries, and tomatoes) and vegetables (such as squash and bell peppers).

Avoid refined foods, such as white breads, pastas, and especially sugar.

Eat fewer red meats and more lean meats, cold-water fish, tofu (soy, if no allergy), or beans for protein.

Use healthy oils in foods, such as olive oil or vegetable oil.

Reduce or eliminate trans fat, found in commercially-baked goods, such as cookies, crackers, cakes, French fries, onion rings, donuts, processed foods, and some margarines.

Avoid alcohol and tobacco.

Drink 6 to 8 glasses of filtered water daily. Exercise at least 30 minutes daily, 5 days a week. A diet with increased refined carbohydrates content leads to an excessive insulin response, which in turn can stimulate the androgen production that contributes to hirsutism and other PCOS symptoms however, increased consumption of omega fatty acids, multivitamins, plant based proteins helps against hirsutism.

\section{Recommended Pugos Supplement}

Astashine and liqimega recommended in $\mathrm{BD}$ dose

Nutrease contain plant based protein is recommended

\section{Nutrition for Heart}

Heart disease, also known as cardiovascular disease, refers to diseases of the blood vessels and heart.

\section{Dietary Recommendations}

\section{Foods to include:}

- Fish and flaxseed contain beneficial omega-3 fatty acids that reduce inflammation and LDL cholesterol, prevent blood clotting, and reduce the risk of heart disease.

- Garlic helps reduce cholesterol, thin the blood, and has antioxidant properties.

- Margarines, salad dressings, and spreads that contain phytostanols (plant substances) help lower cholesterol. 
- Nuts (almonds and walnuts) help lower cholesterol levels. Nuts contain fibre and nutrients such as vitamin E, alpha-linolenic acid, magnesium, potassium, and arginine, which are important for heart health. Although nuts are high in calories, some studies have found that increasing nut consumption by several hundred calories per day does not cause weight gain. Olive oil is a monounsaturated fat that can help reduce blood clots and lower LDL cholesterol.

- Soluble fibre which is found in oats, flaxseed, beans, psyllium, and fruits helps lower cholesterol levels. Insoluble fibre which is found in whole grains, vegetables, and fruits does not lower cholesterol, but studies have shown that it helps protect against heart disease. Aim for a total of $35 \mathrm{~g}$ of fibre daily.

Fruits and vegetables also contain vital antioxidants and studies have shown that those who consume antioxidant-rich diets have lower rates of heart disease. Soy products (tofu, soybeans, miso, and soy protein powder) can help lower cholesterol and triglycerides. Yogurt and fermented milk products have been shown to lower cholesterol levels.

\section{Foods to avoid}

- Foods high in cholesterol should be minimized (organ meats, egg yolks, and whole milk products). Foods high in sugar/refined starches (white flour) raise triglyceride and insulin levels, which increases the risk of heart disease.

- Salt causes water retention and increases the pressure inside your arteries. Reducing salt intake reduces blood pressure in most people. Avoid adding salt to foods and minimize eating processed and fast foods such as deli meats, snacks (chips, pretzels), french fries, and burgers.

- Saturated fat, which is present in animal foods (beef, pork, and dairy) and certain oils (palm oil), can raise cholesterol levels and is associated with increased heart disease risk.

\section{Lifestyle Suggestions}

- Don't smoke, and avoid second-hand smoke, as this increases many risk factors for heart disease.

- Lose excess weight. Losing even 5-10 percent of excess weight can lower cholesterol and blood pressure.

- Exercise regularly. Moderate-intensity activities, such as brisk walking, biking, or swimming, can reduce cholesterol and blood pressure and help with weight management.

- Reduce stress levels. Stress causes the liver to increase the production of cholesterol, which is used to make stress hormones. Try meditation, exercise, or yoga to promote calming and relaxation.

- Manage your blood sugar levels. If you have diabetes or are at risk for diabetes, work on improving your blood sugar levels with exercise and a low-glycemic diet.

\section{Smoking and Heart Disease}

Smoking is a significant risk factor for heart disease as well as cancer. Smoking lowers the good HDL cholesterol, increases blood pressure, and causes serious damage to blood vessels. It is never too late to quit. Within one year of quitting, your risk of heart disease is half of that of a smoker's. 


\section{Recommended Pugos Supplements}

Astashine, Astashine silver, omegaday, liquimega,

\section{Nutrition for Hypothyroidism}

The thyroid gland is located at the base of our neck, it produces two hormones, triiodothyronine (T3) and thyroxine (T4), which circulate through our bloodstream and control metabolic activity in every cell in the body.

\section{Causes}

Hypothyroidism or underactive thyroid occurs when the thyroid gland cannot produce enough thyroid hormones to meet the body's demands. This causes all bodily functions to slow down and we feel tired, sluggish, achy, and gain weight. The most common cause of hypothyroidism was iodine deficiency. Iodine is required for the production of thyroid hormone. The most common cause of hypothyroidism is Hashimoto's disease, which is an autoimmune disorder in which the body makes antibodies that attack the thyroid gland. This impairs the production of thyroid hormone. People with Hashimoto's develop a lump on their thyroid called a goiter.

Hypothyroidism can also result from treatment of Graves' disease (hyperthyroidism) with radioactive iodine, which destroys the thyroid gland, leaving it unable to produce hormones, and from surgical removal of the thyroid gland due to thyroid cancer. A baby can be born without a thyroid gland (congenital hypothyroidism). Diseases of the hypothalamus or pituitary gland can also cause hypothyroidism.

These glands are involved in the regulation of the thyroid gland and the amount of thyroid hormone that is released. The hypothalamus releases thyrotropin-releasing hormone (TRH), which signals your pituitary gland to make thyroid-stimulating hormone (TSH). The amount of TSH released depends on how much T3 and T4 are in our blood. The thyroid gland regulates its production of hormones based on the amount of TSH it receives. Hypothyroidism is easily treated today with thyroid hormones, Nutritional supplements, and various lifestyle approaches.

\section{Sign \& Symptoms of Hypothyroidism}

- Depression, irritability, and anxiety

- Dry, brittle nails

- Dry eyes and droopy eyelids

- Dry, itchy skin; dry hair and hair loss (including eyebrow hair loss)

- Fatigue and sluggishness

- Headaches

- High cholesterol

- Hoarse voice

- Insomnia 
- Joint aching

- Low libido

- Memory loss

- Menstrual irregularities

- Muscle swelling or cramps

- Slow heart rate

- Tingling or numbness in hands and feet

- Weight gain

\section{Risk Factors effecting Hypothyroidism}

- Age: It is most common after age 40

- Family history

- Gender: It is 10 times more common in women

- History of hyperthyroidism, Graves' disease, or thyroid cancer

- Hormone imbalance (high estrogen and cortisol)

- Iodine deficiency

- Medications (lithium, estrogen)

- Poor diet: Lack of iodine or selenium

- Pregnancy: The body produces antibodies that attack the thyroid gland, increasing the risk of miscarriage, premature delivery, pre-eclampsia, and damage to the fetus.

- Stress

\section{Prescription Drugs}

Hypothyroidism is often a chronic problem that requires lifelong treatment. Doctors typically prescribe synthetic thyroid hormone (T4), such as Eltroxin. Blood tests are done to check T3, T4, and TSH levels. Calcium and iron supplements may reduce the absorption of thyroid hormone, so take these products six hours away from your thyroid medication.

\section{Dietary Recommendations}

\section{Foods to include}

- Essential fatty acids are important for proper thyroid function.

- Eat more fish and flaxseed. 
- Sea vegetables such as kelp, nori, dulse, and wakame contain iodine, which is used by the body to make thyroid hormone. Shellfish and saltwater fish also contain iodine.

\section{Foods to avoid}

- Soyabean, Broccoli, Brussels sprouts, cabbage, cauliflower, collard greens, and kale contain goitrogens, which interfere with thyroid hormone synthesis.

- Tap water contains fluorine and chlorine, which can inhibit the body's ability to absorb iodine.

\section{Lifestyle Suggestions}

- Don't smoke, as smoking can worsen hypothyroidism.

- Get regular exercise. Physical activity stimulates the thyroid to secrete more hormones and makes the body more sensitive to any thyroid hormone that is circulating.

- Manage your stress levels. Stress triggers the release of cortisol, which can suppress thyroid function.

\section{Recommended Supplements}

Guggul: Increases production of thyroid hormone (T3). Dosage: $25 \mathrm{mg}$ of guggulsterones (active component) three times daily.

Multivitamin/mineral complex: Many nutrients are required to produce thyroid hormone, such as vitamin C, E, A, and the B-vitamins. Selenium is required for the conversion from T4 to T3.

Many people are deficient in selenium, which may hamper thyroid hormone levels, so a complete multivitamin can ensure that all essential nutrients requirements are met.

Ashwaganda: An herbal product that helps boost thyroid function and also reduces stress.

Dosage: $500 \mathrm{mg}$ three times daily.

Tyrosine: An amino acid involved in the synthesis of thyroid hormone. Dosage: $500 \mathrm{mg}$ twice daily on an empty stomach.

\section{Recommended Pugos Supplements}

Astashine/Astashine gold, liquimega, Optigision gold, Nutrease weight loss therapy.

\section{Nutrition for Hair Loss}

Hair loss is a concern that affects us all, although we hope it doesn't. When we see more hairs than usual in the shower or the hairbrush, we suddenly wonder whether we might be losing our hair. It's comforting to know that the human body sheds approximately 100 of its 100,000-150,000 strands of hair every day and new ones grow to take their place. As we age, this renewal process may slow where more hairs are lost than grown. Real hair loss is most noticeable in men. What is commonly known as male pattern baldness is an inherited condition called androgenetic alopecia and it may begin as early as age 20. Male hair loss is distinguished by a receding hairline or widow's peak and thinning on the crown. 
The rate of hair loss may be slow, gradual, or fast. By age 50, about 50 percent of men will experience thinning and hair loss. For 40-50 percent of women, hair may begin to thin after age 50 (typically after menopause). This is called female-pattern baldness. Women tend to see their hair thin throughout the head, but most visibly on the crown. Significant hair loss for women before age 50 is rare and usually triggered by hormonal fluctuations, stress, or a secondary health concern.

\section{Other kinds of hair loss include:}

Alopecia areata: This form of hair loss is characterized by patchy baldness or bald spots. It affects both men and women equally, both adults and children, but it is rare, affecting less than 2 percent of the population. Hair loss due to alopecia areata is usually triggered by an immune system disorder. Once addressed, the hair usually grows back.

Anagen effluvium: This condition occurs when hair in the growth phase falls out prematurely. Prescription medications used for the treatment of cancer are the most common cause of this condition. Chemotherapy patients may lose up to 90 percent of their hair as a result of anagen effluvium.

Telogen effluvium: A natural part of the hair growth cycle includes a resting phase called telogen, which involves 10 percent of hair at any given time. Telogen effluvium occurs when up to 30 percent of hairs on the head are in the resting phase at any given time. This condition may be caused by physical or emotional stress, and hair growth will return to normal as stress is eased.

The health of your hair is a reflection of the overall state of your health, so it is important to address hair loss from a multipronged approach that includes both the use of standard medical treatments to slow hair loss, and nutritional and lifestyle changes to address and improve health.

- Bald patches

- Scalp irritation

- Sudden, excessive, or increased hair loss

- Visible thinning of the hair around the top and sides of the head.

If hair loss is accompanied by other symptoms such as fatigue, cold hands and feet, dry skin and hair, and menstrual fluctuations, it may be the warning signs of hypothyroidism or an underactive thyroid.

\section{PUT A LEASH ON DHT}

DHT stands for dihydrotestosterone, a hormone formed when the enzyme 5-alpha-reductase breaks down testosterone. DHT is the primary underlying cause of male-pattern baldness. DHT shortens the hair growth cycle, causing the hair follicles to shrink and stop production of hair, leading to hair loss. DHT not only affects men but also women, particularly after menopause when levels of estrogen decrease. Some drugs used to treat baldness work by inhibiting the action of 5-alpha-reductase, thus reducing the formation of DHT. High-fat diets can also boost DHT levels.

- Age: Hair loss is more common with age 
- Burns, injuries, and skin infections such as ringworm

- Drugs used to treat gout, arthritis, depression, heart problems, high blood pressure, and birth control pills can lead to hair loss.

- Genetics

- Hair treatments: Chemicals used for dying, tinting, bleaching, straightening, or perming can cause hair to become damaged and break off if they are overused or used incorrectly; hairstyles that pull your hair too tightly also can cause some hair loss, which is known as traction alopecia.

- Hormonal changes such as pregnancy and menopause

- Immune disorders (lupus, diabetes, thyroid disease)

- Nutritional deficiencies: Inadequate protein, iron, or essential fatty acids

- Severe gastrointestinal disorders

- Stress: Emotional stress, fever, surgery, flu

- Weight problems and extreme dieting

\section{Dietary Recommendations}

Even though hair is not a living tissue, it is important to supply nutrients to the hair follicles in the scalp. While there are no foods that directly stimulate increased hair growth, choose foods that supply the body with a rich supply of vitamins, minerals, antioxidants, fibre, and protein.

\section{Foods to include}

- Ensure adequate protein intake as protein is necessary for hair growth. Choose lean sources of protein (fish, poultry, lean cuts of meat, beans, nuts, seeds, and soy). Meat, poultry, and fish also contain iron, which is required for proper hair growth. Fish and flaxseed contain essential fatty acids necessary for proper hair growth. Nuts and seeds; almonds contain magnesium, which is important for hair growth.

- The outer skin of plants such as potatoes, cucumbers, green and red peppers, and sprouts can strengthen hair because they are rich in the mineral silica.

- Whole grains, vegetables, and fruits are good sources of essential nutrients and fibre.

\section{Foods to avoid}

- Caffeine and alcohol can deplete the body of nutrients and also raise adrenal levels, which can trigger hair loss.

- Foods high in sugar can raise cortisol levels (a stress hormone) and cause the body to produce more androgens, promoting hair loss.

- High intake of salt has been linked to hair loss. Foods high in salt include processed and snack foods, deli meats, and the salt shaker. 
Asian Journal of Applied Science and Technology Volume 4, Issue 3, Pages 215-284, July-September 2020

- Reduce or eliminate pro-inflammatory foods: saturated fat (fatty meats and dairy) and trans fats (processed foods and fried foods). Saturated fat reduces the amount of sex hormone binding globulin (SHBG), a substance that normally binds to testosterone. With less SHBG, more testosterone can be converted into DHT, which promotes hair loss.

\section{Lifestyle Suggestions}

- Regular exercise and healthy sleep habits will increase circulatory and overall health, promoting healthy hair.

- Reduce the frequency of washing and drying your hair. Use a gentle shampoo and conditioner. Avoid using hot water and hair dryers or curling irons whenever possible.

- Avoid exposing hair to chlorinated pool water or any other chemical solutions (perms and dyes).

- Do not over brush your hair. Limit grooming and always be gentle when brushing or combing hair. Keep braids and ponytails loose.

- Give yourself a weekly deep conditioning treatment and scalp massage to protect the hair shaft and stimulate new hair follicle growth.

-Wear a wide-brim hat when outdoors to protect your scalp and hair from the sun's damaging rays.

- Don't smoke. According to one report, smokers were four times more likely to have grey hair than non-smokers and were more prone to hair loss.

B-vitamins: Essential for proper hair growth. A deficiency of the B-vitamins biotin and PABA can cause hair loss. Supplements of biotin may strengthen hair, stimulate new hair growth, slow hair loss, and prevent greying, particularly in those who are deficient in this nutrient. PABA (para-aminobenzoic acid) may protect hair roots and help prevent hair loss. It can also reverse greying in cases where the cause is a deficiency of PABA or other B-vitamins.

Dosage: Take a B-complex vitamin daily.

Essential fatty acids: A deficiency can cause hair loss; supplements can help improve the health of scalp and hair. Take a blend of fish, flaxseed, and evening primrose or borage oil.

Dosage: $2-3$ g daily.

Orthosilicic acid: Enhances collagen formation and makes hair stronger and thicker. One study found that it improved brittleness of hair and nails.

\section{Research Highlight: Essential Oils Stimulate Hair Growth}

A study by Scottish researchers found that essential oils could benefit bald patches caused by alopecia areata. In this study, half of the participants massaged a combination of essential oils of thyme, rosemary, lavender, and cedarwood onto their scalps each day. The other half of participants massaged inactive oils. After seven months, 44 percent of the patients using the essential oils showed significant improvement in hair growth compared to only 15 percent improvement in the placebo group (Archives of Dermatology, 1998: 134; 1349-1352). 
Asian Journal of Applied Science and Technology Volume 4, Issue 3, Pages 215-284, July-September 2020

Grape seed extract: A potent antioxidant that has been shown in preliminary research to stimulate hair growth. Dosage: $50-100 \mathrm{mg}$ daily.

Minerals: Iron, selenium, and zinc are essential for hair growth; a deficiency can cause hair loss. Dosage: Take as part of a daily multivitamin/mineral complex.

Saw palmetto: Used primarily for the treatment of enlarged prostate. It blocks the production of DHT and thus it may be helpful for treating hair loss due to high DHT levels.

\section{Nutrition for Insomnia}

Insomnia is characterized by persistent difficulty falling asleep, waking up too early, awakening frequently during the night, or waking feeling tired and not refreshed. Approximately 30 percent of adults suffer occasionally from insomnia and 10 percent experience chronic insomnia. Sleep is vital for physical and mental health, yet it often gets sacrificed when we are busy. While it is thought that sleep is relaxing and passive, actually quite a lot happens in the body during sleep. During the deepest stages of sleep our bodies' major organs and regulatory systems are busy working on repair and regeneration and secreting certain hormones. The exact amount of sleep needed varies among individuals, but is between seven and nine hours. Getting less than six hours is associated with health problems, such as memory loss, poor concentration, depression, headache, irritability, increased response to stress, high blood pressure, depressed immune function, low libido, and weight gain.

- Daytime fatigue or irritability

- Difficulty in falling asleep or staying asleep

- Waking too early

- Waking feeling tired and fatigued

- Age (the incidence increases with age)

- Diet (high intake of caffeine, refined and processed foods; overeating)

- Exposure to noise and light

- Gender: Women are more likely to suffer insomnia; this may be due to hormonal changes that occur during the menstrual cycle, pregnancy, and menopause.

- Lack of exercise

- Medical conditions (depression, anxiety, cancer, alcoholism, restless legs, hypothyroidism, sleep apnea, bladder problems)

- Medications (blood pressure, antidepressants, decongestants, long-term use of sleeping pills)

\section{HERRY'SATURAL PRESCRIPTION}

- Pain (back pain, headaches, arthritis, fibromyalgia) 
- Poor sleep habits (going to bed at different times)

- Smoking (nicotine is a stimulant)

- Snoring (disrupts deep sleep)

- Stress and muscle tension

- Travelling to a different time zone or working shifts can upset one's internal clock and lead to sleep disturbances.

\section{Dietary Recommendations}

\section{Foods to include}

- A small snack before bed of food that contains tryptophan (an amino acid) stimulates the release of serotonin, a brain chemical that facilitates sleep. Examples include: turkey, soy nuts, or whole-grain crackers or cereal.

- A glass of milk can also promote relaxation, as it contains calcium, which helps promote relaxation.

\section{Foods to avoid}

- Caffeine (coffee, tea, pop, and chocolate) can affect sleep quality, and should be avoided eight hours before bed.

- Alcohol may help you fall asleep, but it causes nighttime wakening and reduces sleep quality, so minimize or avoid completely.

- Go easy on sugary foods, especially in the evening, as they can cause a sugar rush and affect your ability to fall asleep.

\section{Lifestyle Suggestions}

- Set aside at least seven to eight hours for sleep. Leaving only five or six hours may make you feel stressed and affect your ability to fall asleep.

- Establish a regular bedtime and wake time.

- Do relaxing activities before bed—read a book, have a warm bath, or meditate.

- Reserve your bedroom for intimacy and sleep only; don’t work in your bedroom.

- Make your bedroom dark, quiet, and comfortable.

- Exercise regularly early in the day.

- Don't smoke, as nicotine is a stimulant and impairs your ability to fall asleep and have a restful sleep.

- Consider massage, yoga, or meditation for relaxation.

\section{PUGOS RECOMMENDED NUTRITION}

FOR MEN: M-Rush, Optigision gold,

FOR WOMEN: Onemincal, Optigision gold, 


\section{AJAST}

Asian Journal of Applied Science and Technology

Volume 4, Issue 3, Pages 215-284, July-September 2020

\section{Nutrition to Remove Kidney Stones}

The kidneys are two bean-shaped organs, about the size of your fist, that are located behind your abdomen on each side of your spine. The main function of the kidneys is to remove excess fluid and wastes from your blood in the form of urine. Kidney stones, known medically as renal lithiasis, occur when minerals and other substances in the urine form crystals inside your kidneys.

Over time, these crystals may combine to form a small, hard mass or stone.

\section{Dietary Recommendations}

\section{Foods to include}

- Apples, green leafy vegetables, kelp, seeds, legumes, and figs are good sources of magnesium, and a magnesium deficiency can increase the risk of stones.

\section{Kidney Stones}

- Drink lots of water to help flush out your kidneys and prevent dehydration.

Add some lemon slices or juice to your water. Lemon contains citric acid, which acidifies the urine and can help assist the passage of calcium oxalate stones.

- Eat lots of vegetables, fruits, and whole grains. Fish, beans, seeds, and poultry provide good sources of protein.

- Oranges, bananas, raisins, avocado, and artichokes contain potassium, which reduces urinary calcium excretion. Boosting intake of potassium has been shown to reduce the risk of stones.

- Pumpkin seeds have been shown in preliminary research to reduce the risk of stone formation.

\section{Foods to avoid}

- Foods high in oxalic acid increase the risk of stone formation, so minimize intake of spinach, rhubarb, beet greens, nuts, chocolate, tea, bran, almonds, peanuts, and strawberries.

Other foods contain some oxalic acid, but only these have been found to be a problem.

- Grape fruit juice has been linked to increased risk of kidney stones.

- Studies have shown that a diet high in animal protein increases the risk of stone formation.

Animal protein increases the excretion of calcium, causing a buildup of calcium in the urine.

- Reduce sodium intake as a high sodium intake can promote calcium excretion and also lead to dehydration.

Limit sodium to 2,000 mg daily. Foods high in sodium include snack foods, deli meats, condiments, and processed foods. Avoid using the salt shaker.

- Sugar has been linked to increased risk of oxalate stones. Limit intake of sweets, candy, and soft drinks.

Soft drinks also contain phosphoric acid, which also increases the risk of stones. 


\section{Lifestyle Suggestions}

- Get regular exercise.

- Have a warm bath with mineral salts and add a few drops of lavender or chamomile oil to promote relaxation and ease pain.

\section{Calcium Supplements and Kidney Stones}

For years, those with or at risk of calcium oxalate stones were told to avoid calcium rich foods and supplements. However, recent studies have shown that high-calcium diets actually help prevent stones because dietary calcium binds with oxalates in the gastrointestinal tract so that oxalates can't be absorbed from the intestine and excreted by the kidney to form stones. Calcium supplements seem to have the same protective effect as dietary calcium, but only if they're taken with meals. When taken on an empty stomach, the calcium can't bind with the oxalates in food, thus increasing the risk of stone formation.

\section{Recommended Pugos Supplements}

Astashine silver, Optigision gold, liquimega

\section{Nutrition for Low Libido in Men \& Women}

Low libido is an absence or deficiency of sexual fantasy and desire for sexual activity.

This is also referred to as inhibited sexual desire. It is difficult to define "normal" sexual desire as it varies among individuals, gender, and age. However, surveys have found that approximately 30-40 percent of all adults complain of a low sex drive.

This may be a primary condition (a person never felt much sexual desire) or secondary (a person used to possess sexual desire, but no longer has an interest).

There are many factors that affect one's libido—psychological, physical, medical, and even lifestyle.

There are many potential causes and risk factors for low libido, including:

- Health conditions: Cushing's syndrome, fatigue, menopause, postpartum depression, diabetes, hysterectomy, and obesity

- Hormonal imbalance: Low thyroid, estrogen, testosterone, or DHEA

- Nutritional deficiency in zinc

- Physical problems: Vaginal dryness, vulvodynia, impotence (ED), and inability to reach climax

- Prescription drugs: Beta-blockers (for blood pressure), birth control pills, antidepressants, tranquilizers, Proscar (for prostate enlargement), Tamoxifen (for breast cancer)

- Psychological issues: Stress, depression, relationship conflict, negative or traumatic sexual experiences

- Use of alcohol or marijuana 
Asian Journal of Applied Science and Technology

Volume 4, Issue 3, Pages 215-284, July-September 2020

Low libido affects more women than men. This could be due to hormonal changes (PMS, child birth, and menopause).

\section{Sherry's Natural Prescription}

\section{Dietary Recommendations}

\section{Foods to include}

- Eat a healthy, whole foods diet, seasonal fruits, Vegetables. Oysters are high in zinc, which raises sperm and testosterone production.

\section{Foods to avoid}

- Refined carbohydrates, sugar, processed and fast foods, and caffeine can trigger mood swings, irritability, and anxiety.

- Saturated and trans fats (red meat, high-fat dairy, deep-fried and processed foods) can impair blood vessel health.

- Alcohol may reduce inhibitions; however, it can also act as a depressant and negatively affect sexual function.

\section{Lifestyle Suggestions}

- Exercise: Aerobic activities such as walking and cycling can reduce stress, improve mood, increase energy, and improve circulation (improved blood supply to the pelvic area may help to improve sexual sensation and satisfaction).

- Maintain a healthy body weight. Women with vaginal dryness can try a lubricant such as Replens or Astroglide.

- Don't smoke as smoking causes damage to nerves and blood vessels, which can affect sensation.

- Relax - try massage, meditation, yoga, breathing exercises, or a warm bath. Aromatherapy oils known to inspire romance include rose, clary sage, sandalwood, and jasmine.

- Set aside time to be intimate with your partner and work on improving communication.

\section{Recommended Pugos Nutrition Supplements}

FOR MEN: M-Rush, Optigision gold, Astashine silver

FOR WOMEN: Onemincal, Optigision gold, Astashine silver

\section{Nutrition for Menopause}

Menopause is a natural phase in a woman's life that can be thought of as a time of ovarian retirement. At birth women have about one million eggs in their ovaries. At puberty ovulation starts and eggs are released by the ovaries each month for the purpose of conception. As the years go by, the amount of eggs gradually declines until menopause, when the ovaries shut down and stop producing estrogen and progesterone, the two main female sex hormones. Menopause occurs when ovulation ceases and a woman can no longer conceive naturally. After menopause the adrenal glands, which supply some sex hormones throughout life, become the primary source. 
Asian Journal of Applied Science and Technology Volume 4, Issue 3, Pages 215-284, July-September 2020

Women who have poor adrenal function, which can be caused by chronic stress, poor diet, lack of sleep, or excessive caffeine, are not able to provide adequate hormone amounts, and may have more severe menopausal symptoms. Estrogen can also be produced in the fat cells from androgens. There are many lifestyle measures and supplements that can promote hormone balance and ease menopausal concerns.

\section{Are You In Menopause?}

The milestone of menopause is reached when a woman goes one year without a menstrual period. The average age for menopause is about 51 years, but it can occur naturally between ages 40 and 55 . The decade or so before menopause is called perimenopause. During this time hormone levels fluctuate and the menstrual cycle becomes erratic. Menopausal symptoms are caused primarily by an imbalance of the hormones estrogen, progesterone, and/or testosterone. High cortisol levels caused by stress can negatively impact these hormones as well. The severity and duration of symptoms experienced vary due to genetics, ethnicity, cultural factors, and even attitude.

\section{They include:}

- Fatigue, foggy head, headache, memory loss

- Fluid retention and weight gain

- Hot fl ashes and night sweats

- Incontinence, bladder infections, Low libido

- Mood swings, irritability, anxiety, depression

- Vaginal dryness

\section{Menopause and Weight Gain}

There are several factors that can contribute to weight gain in menopause. High estrogen levels, as is common in perimenopause or among those with estrogen.

\section{Dietary Recommendations}

\section{Foods to include:}

- Vegetables, fruit, whole grains, and legumes provide essential vitamins, minerals, antioxidants, fibre, and compounds that can reduce the risk of cancer and heart disease and improve overall health. In particular, load up on broccoli, Brussels sprouts, cauliflower, and cabbage, as these vegetables contain compounds that help the liver process hormones while reducing the risk of breast cancer.

- Soy foods (tofu, soy milk, soybeans, and soy nuts) contain isoflavones (plant based estrogens), which help minimize menopausal symptoms, offer protection against breast cancer, and improve bone health.

- Flaxseed is a rich source of fibre, which promotes bowel regularity and reduces the risk of colon and breast cancer and lowers cholesterol. It also contains lignans (another form of phytoestrogen), which may help balance estrogens and reduce menopausal symptoms. 
- Fish, nuts, and seeds contain essential fatty acids that are important for heart and skin health.

\section{Foods to avoid}

- Alcohol, caffeine, and spicy foods can worsen hot fl ashes.

- Saturated fat (red meat) and trans fat (processed, deep-fried, and fast foods) increase the risk of heart disease.

\section{Lifestyle Suggestions}

- Stress can make menopausal symptoms more pronounced and affect adrenal gland function, reducing hormone production. Meditation, yoga, and breathing techniques can help reduce stress. Massage and acupuncture promote relaxation and studies have shown that acupuncture can reduce hot flashes.

- Get regular exercise. Studies have shown that regular exercise reduces the frequency and severity of hot flashes. It also improves mood and sleep, protects against heart disease, and weight-bearing activities strengthen the bones.

- Don't smoke. Smoking can worsen hot flashes and symptoms of anxiety, irritability, and depression.

- Consider a support group: it may help to share your experience with others.

- Laughter is good medicine. Watch funny movies, go to comedy shows, and spend time with people who make you happy and laugh. Ensure adequate calcium intake or take a mineral supplement for bone health.

\section{Recommended Pugos Supplement}

Onemincal, liquimega, Astashine Silver.

\section{Nutrition for Obesity}

Obesity has become one of the greatest health threats, as it is linked to many serious diseases that result in premature death. Over two-thirds of adults are overweight and nearly one-quarter obese.

Children, and adolescents are becoming increasingly overweight and obese, resulting in diseases and a predicted shortened life expectancy. There are several methods used to determine whether a person is overweight or obese. The most commonly used method is the body mass index (BMI), which compares our height and weight to a standard. To calculate your BMI, take your weight in kilograms and divide that by your height in metres squared $(\mathrm{Kg} / \mathrm{m} 2)$. A BMI between 18.9 and 24.9 is considered normal, a BMI between 25 and 29.9 is classified as overweight and a BMI greater than 30 indicates obesity. Studies have shown that the greater the BMI, the greater the risk of developing health problems, such as heart disease and diabetes.

Men with greater than 25 percent, and women with more than 30 percent, body fat are considered obese and at significant risk of developing health problems.

Recommended ranges of body fat are 15-25 percent for women and 10-20 percent for men.

Numerous studies have found that those who maintain a lean body live longer, suffer less disease, and enjoy a better quality of life. If you are overweight or obese, it is important to know that even small losses can improve your 
Asian Journal of Applied Science and Technology

Volume 4, Issue 3, Pages 215-284, July-September 2020

health. Studies have shown that losing 10-15 percent of excess weight can reduce blood pressure, blood sugar, cholesterol, and triglycerides.

\section{Factors effecting obesity}

Obesity also increases one's risk of breathing disorders, gall bladder disease, sexual dysfunction, osteoarthritis, and stroke. The emotional consequences—-low self-esteem, depression, and anxiety—can be just as serious.

- Excess calorie intake; poor dietary choices

- Genetics

- Hormonal imbalances (high insulin; low thyroid, testosterone, DHEA, or growth hormone)

- Lack of physical activity

- Lack of sleep (increases appetite and promotes food cravings)

- Low metabolic rate (rate at which calories are burned at rest)

- Medications (antidepressants and hormones)

- Stress (causes hormonal alterations that increase appetite and reduce metabolism)

\section{Dietary Recommendations}

Avoid fat diets.

A detoxification program can eliminate stored toxins and help the body recover from toxins.

\section{Foods to include}

- Quality proteins, such as lean meat, poultry, fish, beans, soy products, nuts, and seeds are essential for building and maintaining muscle mass, which drives our metabolism. The recommended amount of protein is based on body weight and activity level. For the average person, a daily intake of 0.8 to $1 \mathrm{~g}$ per kilogram, or $0.5 \mathrm{~g}$ per pound of body weight is adequate. Body-builders and those involved in intense exercise require more protein.

- Nutrient-dense, low-glycemic carbohydrates such as fresh fruits, vegetables, legumes (beans, peas, and lentils) and whole grains (multigrain breads, brown rice/pasta, oats, and flaxseed) are high in fibre, which fills us up and aids digestion and elimination. Fibre also balances blood sugar and insulin levels, which is important in regulating appetite and energy levels. Aim for five to 10 servings of fruits, vegetables, and legumes and two servings of grains daily.

- Healthy fats such as those found in cold-water fish, olive oil, nuts, and seeds provide us with essential fatty acids (healthy fats), which are essential for health and disease prevention.

\section{Health Tip}

Eat five times daily: three small meals and two snacks between meals. This will help keep metabolism and energy levels high. 
Asian Journal of Applied Science and Technology

Volume 4, Issue 3, Pages 215-284, July-September 2020

Do not skip meals as this can increase appetite, reduce metabolism, and lead to binge eating.

\section{Foods to avoid}

- Processed and fast foods, candy, cookies, and sweets are high in sugar and fat calories and low in nutritional value. Limit refined grains (white bread/pasta/rice) as these foods are broken down quickly into sugar, causing fluctuations in blood sugar and insulin levels, which can increase appetite and promote fat storage. Avoid saturated and hydrogenated fats, which are high in calories, promote weight gain, and are linked to heart disease and cancer. Reduce alcohol intake as these drinks are high in empty calories. Cut down on salt as using the salt-shaker and eating salty snack foods and processed foods can cause fluid retention.

\section{Lifestyle Suggestions}

- Boost your activity level: Regular physical activity is essential to achieving a healthy body weight.

Aim for 30 minutes to one hour of moderate-intensity activity daily.

Do a combination of:

- Cardiovascular activities such as walking, cycling, and swimming, which promote fat loss by burning calories and stored fat.

- Resistance activities (working out with weights, exercise bands/tubes, or machines) help to build lean muscle mass, which will elevate your metabolic rate so that you burn more calories.

- Stretching promotes flexibility and relaxation, and reduces injuries. Spend at least five minutes stretching your muscles after a workout.

\section{Obesity}

- Reduce stress, which can trigger appetite and food cravings and increases the production of cortisol, a hormone that promotes fat storage around the abdomen.

- Get adequate sleep as lack of sleep increases production of the hormone ghrlin, which stimulates appetite and can also reduce production of growth hormone, an important regulator of our metabolism.

\section{Recommended Pugos Supplements}

\section{ASTASHINE, LIQUIMEGA, NUTREASE}

Regular Detoxification of Body with Nutrease helps to Prevent Obesity.

Nutrease weight loss program is helpful in Obese patients as it is natural plant based diet

\section{Nutrition for Osteoarthritis}

Osteoarthritis (OA), also known as degenerative joint disease, is the most common form of arthritis, the disease is caused by wear and tear on the joints and deterioration of joint cartilage, which is a gel-like material that covers and protects the ends of bones. As the cartilage breaks down, we lose the cushion that it provides between our bones. 
As a result, the bones start to rub against each other, causing a grinding or clicking of the joints, bone damage, inflammation, and pain. As the disease progresses, the pain can become severe and affect one's mobility and quality of life. The disease can affect any joint, but it is most common in weight-bearing joints such as the knees, hips, ankles, fingers, neck, and spine, and it causes:

- Grinding or clicking in the joints

- Growths (spurs)

- Joint stiffness and pain in the morning or after exercise

- Pain

- Red, warm, swollen joints

- Reduced mobility

In some people, the symptoms remain mild or even go away at times. In others the condition progresses as the joint deteriorates and inflammation sets in. Cold weather and humidity can worsen the symptoms.

- Age: It is more common in those over 45 , but can occur at any age

- Family history: Inheriting abnormal joint or cartilage structure

- Obesity: Excess weight puts stress on joints, especially hips and knees

- Injury: Joint damage due to an accident, sports, or occupation (i.e., carpet laying)

- Having another forms of arthritis, such as rheumatoid Osteoarthritis

\section{Dietary Recommendations}

\section{Foods to include}

- Asparagus, cabbage, garlic, and onions contain sulphur, which is beneficial for the joints.

- Cold-water fish, olive oil, flaxseed, and hemp are rich in essential fatty acids and can help reduce inflammation.

- Green tea: Several studies have found that the antioxidants in green tea (catechins) can reduce chronic joint inflammation and slow cartilage breakdown.

- Pineapple contains the enzyme bromelain, which can help reduce inflammation.

\section{Foods to avoid}

- Nightshade vegetables (potatoes, tomatoes, peppers, eggplant) contain a substance called solanine, which can trigger pain and inflammation; preliminary studies have found benefits in avoiding these foods.

- Processed, fast, and deep-fried foods contain trans-fatty acids, which trigger inflammation.

- Red meat, milk, cheese, and deep-fried foods contain saturated fat, which triggers inflammation.

- Sugar, refined carbohydrates, alcohol, and caffeine 


\section{Lifestyle Suggestions}

- Acupuncture offers short-term benefits for pain relief.

- Apply ice or heat packs to the affected joints. Heat relaxes aching muscles and reduces joint pain. Cold helps to reduce inflammation and pain.

- Assistive devices, such as canes, walkers, and joint supports, reduce joint strain.

- Avoid activities that put excess strain on the joints (running, jumping, kneeling, or standing for long periods).

Take a load off and rest when doing heavy or repeated tasks such as gardening, shovelling snow, or doing housework.

- Exercise helps reduce pain and prevent further joint damage; strengthens muscles and bones, which will help improve mobility and function; and also helps with weight management.

- Light massage with botanical oils (camphor, eucalyptus, pine needle, or rosemary) can help reduce pain and promote relaxation.

\section{Top Recommended Supplements}

Chondroitin: A component of human cartilage, bone, and tendon. It enhances the shock absorbing properties of collagen and blocks enzymes that break down cartilage. Studies have shown that it reduces pain and inflammation, improves joint function, and reduces the need for NSAIDs. It may also slow the progression of OA. Chondroitin is often combined with glucosamine. It may take several months to work. Dosage: 1,200 mg daily; well tolerated.

Glucosamine: A major component of joint cartilage that provides building blocks for growth, repair, and maintenance of cartilage; helps cartilage absorb water; and keeps joints lubricated. Numerous studies have found glucosamine comparable to NSAIDs, yet better tolerated. It may take six to eight weeks to notice benefits. Avoid if allergic to shellfish. Dosage: $1,500 \mathrm{mg}$ daily.

S-adenosylmethionine (SAMe): A nutrient involved in cartilage formation and repair. It reduces pain and inflammation and stimulates cartilage formation. Numerous studies have found it equally effective as NSAIDs with no major side effects. Dosage: 400-1,200 mg daily.

Boswellia: A tree resin used in traditional Indian medicine; some studies show that it reduces pain and inflammation similarly to NSAIDS, but it does not cause stomach irritation. Dosage: $400 \mathrm{mg}$ three times daily.

Bromelain: An enzyme with anti-inflammatory activity that improves joint mobility. It is well tolerated; however, it may thin the blood, so use cautiously along with blood-thinning medications. Dosage: 2,000-6,000 MCU daily.

Capsaicin: A hot pepper extract that reduces pain. It is available in creams; look for a product with $0.025-0.075$ percent capsaicin (Zostrix or Menthacin); wash hands after application to avoid getting in the eyes.

Curcumin: An antioxidant that has been shown in studies to have anti-inflammatory effects comparable to cortisone. Dosage: $400 \mathrm{mg}$ three times daily; no side effects. 
Asian Journal of Applied Science and Technology

Volume 4, Issue 3, Pages 215-284, July-September 2020

Essential fatty acids: Both the omega-3s (fish oil) and omega-6s (borage, primrose oil) help to reduce the pain and inflammation and lubricate the joints.

\section{Recommended Pugos Supplements}

FLEXAGOLD, OMEGADAY, LIQUIMEGA

\section{Nutrition for Prostate Enlargement (Benign Prostatic Hyperplasia)}

Benign prostatic hyperplasia (BPH) is a non-malignant enlargement of the prostate gland, which is located just below a man's bladder, surrounding the urethra (the tube that drains urine from the bladder). This gland produces seminal fluid, which nourishes and transports sperm.

About half of men at age 50 have prostate enlargement and up to 90 percent of men in their seventies and eighties are affected. At birth, the gland is about the size of a pea. In a man, it is the size of a walnut and stays this size until his late forties when it undergoes a growth phase. The cells in the gland begin to reproduce more quickly, which causes the gland to enlarge.

As it enlarges, it compresses the urethra and partially blocks the flow of urine. Prostate enlargement is not related to prostate cancer, but it can cause distressing urinary symptoms, such as difficulty in starting or stopping a stream of urine and the need to void frequently.

The cause of prostate enlargement is believed to be hormonal changes associated with aging. As men age, testosterone levels decline and estrogen levels increase.

Levels of another hormone called dihydrotestosterone (DHT) increase. These changes are implicated in triggering growth of the prostate, so treatments for BPH are geared toward correcting the hormonal imbalances.

The signs and symptoms of prostate enlargement vary greatly among men.

- Age: It is most common in men over age 60

- Diet: High intake of saturated fat and low intake of fibre

- Ethnicity: It is more common in Caucasian men than in Asian men

- Family history

\section{Sherry's Natural Prescriptionp}

\section{Dietary Recommendations}

\section{Foods to include}

- Drink plenty of water to keep fluid through your urinary tract.

- Fish and flaxseed contain beneficial fatty acids that reduce inflammation.

- Foods rich in soy, such as tofu, contain substances (isoflavones) that may also protect the prostate and ward off cancer. 
- Pumpkin seeds contain zinc, an essential nutrient for the prostate.

- Tomatoes contain lycopene, a nutrient that has protective effects on the prostate.

\section{Foods to avoid}

- Alcohol increases urine production and irritates your bladder.

- Caffeine will increase urine production, cause bladder irritation, and aggravate

Prostate Enlargement (Benign Prostatic Hyperplasia)

- Saturated fat (red meat and dairy) can worsen symptoms.

- Sugar hampers immune function and may increase the risk of bladder infections. Limit candy, soft drinks, and sweets.

Limit drinking after 7 p.m. to reduce your need to go to the bathroom during the night.

\section{Lifestyle Suggestions}

- Exercise regularly. Studies show that men who are active have fewer symptoms of BPH.

- Empty your bladder regularly.

\section{Recommended Pugos Nutrition Supplements}

Astashine, M-Rush, liquimega

\section{Nutrition for Psoriasis}

Psoriasis is a common and recurring condition that affects the life cycle of skin cells.

Normally, it takes about a month for our skin cells to move from the lowest skin layer (where they're produced) to the outermost layer (where they die and flake off). In people with psoriasis, the entire life cycle takes only days. With rapid cell growth, the skin cells build up, forming thick, silvery scales and itchy, dry, red patches that are sometimes painful. This is considered a chronic condition as there are persistent periods of remission and then flare-ups.

It is thought that psoriasis is caused by a disorder of the immune system.

The overactive T cells (a type of white blood cell) attack healthy skin cells, causing an increased production of skin cells and other immune responses that lead to an ongoing cycle of rapid skin turnover. Dead skin and white blood cells can't slough off quickly enough and build up in thick, scaly patches on the skin's surface.

\section{Dietary Recommendations}

\section{Foods to include}

- Essential fatty acids help to reduce inflammation. Eat more cold-water fish (salmon, herring, mackerel, and trout). Flaxseed and flaxseed oil are also good sources of EFAs. 
- Fibre-rich foods (whole grains, vegetables, and fruits) aid detoxification.

- Orange, yellow, and green vegetables contain vitamin A, which may be deficient in those with psoriasis.

- Pumpkin seeds provide zinc, which is often deficient in those with psoriasis.

\section{Foods to avoid}

- Alcohol can cause flare-ups and also decrease the effectiveness of treatments.

- Gluten, a protein present in wheat, rye and barley, and triticale, can be a trigger for some people.

- Saturated fat (red meat and dairy products) may worsen inflammation.

\section{Lifestyle Suggestions}

- Work on identifying and avoiding your triggers. Stress, smoking, and food sensitivities are common triggers.

- Bathe daily in warm water to remove scales and calm inflamed skin. Add mineral or sea salts, bath oil (lavender), or oatmeal to the water and soak for at least 15 minutes. Avoid hot water and harsh soaps, which can make your symptoms worse. Apply a moisturizer after bathing and reapply as needed. Use a heavy cream or an ointment as they will keep the skin hydrated longer than lotions.

- Do not scratch, pick, or rub at the lesions; this will only make it worse.

- Spend some time in the sun. We produce a powerful form of vitamin D when our skin is exposed to sunlight and this vitamin D can improve skin lesions. However, too much sun (getting sunburn) can worsen the problem. Try 10-15 minutes in direct sunlight. When spending a longer time outdoors, apply a sunscreen to areas not affected with psoriasis to protect against sunburn.

\section{Recommended Pugos Supplements}

Astashine, liquimega, Nutrease

\section{Arthritis (Rheumatoid)}

"Arthritis" is a term used to describe conditions that cause joint inflammation. There are over 100 forms of arthritis. Rheumatoid arthritis (RA) is the second most common form of arthritis and affects one in 100 persons.

RA is an autoimmune disease. The immune system produces antibodies that attack healthy joints, causing inflammation in the lining of the joints and pain. Chronic, uncontrolled inflammation leads to damage and destruction of the cartilage and joint tissues (bones, tendons, and ligaments) and the formation of scar tissue. Those with severe RA often suffer with deformity, reduced joint mobility, and pain that limit normal daily activities.

There are several lifestyle modifications and supplements that can help ease the pain and inflammation of RA and improve quality of life.

The symptoms of RA vary from person to person, but typically affect the fingers, wrists, knees, ankles, and toes. It may start gradually or as a sudden, severe attack causing: 
- Flu-like symptoms (fatigue, weakness, and loss of appetite)

- Joint pain, stiffness, and warmth

- Swollen, red joints

Some people have continuous symptoms and others go through periods of remission followed by flare-ups. Severe cases of RA can cause red skin lumps (rheumatoid nodules) on the elbows, ears, nose, knees, toes, or scalp and deformity of the joints.

- Age: It can strike at any age; however, most people develop it between ages 25 and 50.

- Gender: Women are three times more likely than men.

- Family history: This may increase risk, but is not absolute; some people with a family history never get the disease, and others with no history will get it.

- Smoking cigarettes over a long period of time

- Environment: Infection (bacterial or viral), stress, food allergies, or toxin exposure may trigger the onset in those who are susceptible.

Non-steroidal anti-inflammatory drugs (NSAIDs), such as ibuprofen, naproxen, and indomethacin, are commonly prescribed to reduce pain and inflammation, yet their popularity is waning as many serious side effects have emerged. Ironically, long-term use of these drugs can worsen joint health by accelerating the breakdown of cartilage. Corticosteroids such as prednisone are used to reduce pain and inflammation, but are not recommended for long-term use as they can cause bruising, thinning of bones, cataracts, weight gain, a round face, and diabetes.

Disease modifiers such as methotrexate, Plaquenil, and Imuran are taken to halt inflammation, slow the progression of RA, and control the immune system response. These drugs can cause serious side effects, such as nutrient deficiencies, blood disorders, and increased risk of infection. Surgery to remove inflamed tissue and replace joints may be done for severe cases.

CAUTION: NSAIDs can cause serious and life-threatening side effects such as stomach ulcers and bleeding, increased gut permeability, water retention, and kidney damage, increased risk of heart attacks.

\section{Dietary Recommendations}

\section{Foods to include}

- Cold-water fish, olive oil, flaxseed, and hemp, which are rich in essential fatty acids, can help reduce inflammation.

- Asparagus, cabbage, garlic, and onions contain sulphur, which is beneficial for the joints.

- Pineapple contains the enzyme bromelain, which can help reduce inflammation.

- Green tea: Several studies have found that the antioxidants in green tea (catechins) can reduce chronic joint inflammation and slow cartilage breakdown. 
Fish oil: Contains beneficial omega-3 fatty acids (EPA and DHA) which have been shown in studies to reduce the pain and inflammation associated with arthritis. These "good" fats work in part by boosting levels of prostaglandins, which are hormone-like substances that have anti-inflammatory activity.

Multivitamin and minerals: Studies have found certain nutrients are depleted in those with RA, including antioxidants, B-vitamins, copper, selenium, and zinc.

These nutrients help reduce free radical damage and inflammation and support cartilage, bone, and joint health.

Curcumin: A spice from the turmeric plant, curcumin is an antioxidant and has been shown in studies to have anti-inflammatory effects comparable to cortisone; no side effects.

\section{Foods to avoid}

- Nightshade vegetables (potatoes, tomatoes, peppers, eggplant) contain a substance called solanine, which can trigger pain and inflammation; preliminary studies have found benefits in avoiding these foods.

- Processed, fast, and deep-fried foods contain trans-fatty acids, which trigger inflammation.

- Red meat, milk, cheese, and deep-fried foods contain saturated fats, which trigger inflammation.

- Sugar, refined carbohydrates, alcohol, and caffeine may worsen symptoms.

\section{Lifestyle Suggestions}

- Acupuncture reduces pain and stress. Physical therapy, such as ultrasound and laser therapy, is beneficial for pain and inflammation. Exercise: Light activities that are not stressful on the joints, such as swimming, cycling, and stretching (yoga), help to improve joint mobility and overall health.

- Hot paraffin baths, therapeutic mud treatments, baths with Epsom/mineral salts, and sauna can reduce pain and inflammation. Apply hot or cold compresses to the affected joints. Both are helpful.

- Light massage with botanical oils (camphor, eucalyptus, pine needle or rosemary) can help reduce pain and promote relaxation.

- Assistive devices, such as canes, walkers, and joint supports, offer support and reduce joint strain.

\section{Recommended Pugos Nutrition}

Flexagold, liquimega, astashine, curcumet

\section{Nutrition for sciatica}

Sciatica refers to pain that radiates along the path of the sciatic nerve, which branches from your lower back through your hips and buttocks and down each leg. Typically, sciatica affects only one side of your body.

Sciatica most commonly occurs when a herniated disk, bone spur on the spine or narrowing of the spine (spinal stenosis) compresses part of the nerve.

This causes inflammation, pain and often some numbness in the affected leg. 


\section{Foods to be taken in sciatica}

Magnesium-rich foods include dairy products, fish, meat, seafood, apples, apricots, brown rice, beans etc.

Foods containing significant amounts of vitamin B12 like liver, oysters, lamb and cheese also might be beneficial in treating your sciatic nerve pain.

Include foods rich in omega 3 fatty acids, which include salmon, sardines, herrings and mackerel etc.

Vitamins A, such as milk, cheese and curd, carrots, dark green leafy vegetables, orange-colored fruits, e.g. mangoes and apricots, fortified margarine, eggs, mackerel and other oily fish.

Vitamin $\mathrm{C}$ is found in fruits especially citrus, regular and sweet potatoes, cabbage, spinach broccoli, tomatoes, and green and yellow vegetables.

Adequate Potassium is equally important in managing sciatica as it provides toughness to the muscles and helps in neurotransmission, food rich in potassium are white beans, dark leafy greens, potatoes, apricots, avocados, mushrooms, fish (salmon) and Banana.

Fiber foods

\section{Foods to be avoided}

All the flour and refined sugars that terribly wear nervous system.

Don't drink cow's milk and eat derivatives, fried sausages and processed or junk food.

Yoga and Exercise

\section{Stretch exercises}

Knee to chest stretch

Sciatic mobilizing stretch

Back extensions

Standing hamstring stretch

Lying deep gluteal stretch

Ice packs, or a simple package of frozen peas wrapped in a towel, can work wonders to provide instant relief for sciatica. Apply the packs directly on the affected area for 20 minutes, every two hours, until the pain is completely gone.

Ice packs are a proven way to get instant relief, but the sciatic nerve is located deep in the body, so the packs cannot go deeper to where the inflammation is located. Apply a hot pack, right after the ice pack or better yet, take a hot bath. By alternating the temperatures, you can boost the circulation and lymph flow. This will reduce the deeper inflammation and aid in the healing process. For even better results, add some Epsom salts or anti-inflammatory herbs or essential oils to your bath. 
Asian Journal of Applied Science and Technology

Volume 4, Issue 3, Pages 215-284, July-September 2020

Deeper messaging or trigger-point therapies have shown great success in the treatment of muscle spasms, pain, and numbness in the legs and toes. Herb-infused oils and essential oils will also give you good results.

Last, but not least, try to get enough sleep. In doing so, this will give your nerves and body time to relax, heal and balance. Extra sleep and rest will help rebuild and strengthen your nerves.

Avoid constipation

\section{Suggested pugos nutrition}

A synergistic effect of Liquimega, optigision gold and flexagold is recommended

\section{Nutrition for UTI Infections}

Vaginitis is an inflammation of the vagina that can cause discharge, itching, and pain.

Vaginitis often results from irritation, infection, or hormonal changes, such as low estrogen levels in menopause. Most women will experience vaginitis at some point in their lives, and while it is unpleasant, it can be dealt with effectively with both conventional and natural approaches.

There are many different types of bacteria and yeast normally present in the vagina.

However, when the numbers of these organisms become out of balance, infection can occur, causing vaginitis.

The most common cause of vaginitis is an overgrowth of the naturally occurring yeast Candida albicans.

An estimated three out of four women will have a yeast infection in their lifetime.

Vaginitis can also result from reduced estrogen levels after menopause, which causes the vagina to become thinner and drier, which may lead to itching, burning, or pain. Use of vaginal sprays, douches, perfumed soaps, and spermicidal products can also cause vaginal irritation, burning, and itching.

- Change in your normal vaginal discharge and odour

- Light vaginal bleeding

- Pain during intercourse or urination

- Vaginal itching or irritation

Bacterial vaginosis typically causes a greyish-white, fishy-smelling discharge. Yeast infections cause a thick, white discharge that resembles cottage cheese. Trichomoniasis causes a greenish-yellow, sometimes frothy discharge in women. Men have itching, burning, or pain Symptoms in UTI Infections.

\section{Dietary Recommendations}

\section{Foods to include}

- Eat natural (organic) yogurt with live cultures daily. Studies have shown that daily consumption of yogurt can reduce the occurrence of $\mathrm{BV}$ and yeast infections.

- Garlic and onions have antifungal properties. 


\section{Foods to avoid}

- Alcohol, aged cheese, and fermented foods (vinegar and soy sauce) contain yeast and moulds, which can be a problem.

- Sugar allows yeast to thrive, and can increase the likelihood of yeast infections in susceptible women. Cut down on all sugar-containing foods.

Food allergies are believed to be a contributory factor in some cases of recurrent irritant vaginitis.

\section{Lifestyle Suggestions}

- Wear cotton underwear and minimize or avoid wearing pantyhose. Do not wear pants that are tight at the crotch.

- Do not use deodorized products, such as tampons, douches, and feminine deodorant sprays.

- Wash the vaginal area with a mild, unscented soap.

\section{Recommended Pugos Nutrition}

UTI IN FEMALE: Astashine, Curcumet, Optigision gold

UTI IN MALE: Astashine, M-Rush, Curcumet

\section{Nutrition for Varicose Veins}

Varicose veins are dilated, bulging, discoloured veins that you can clearly see through the skin. It is estimated that at least 15 percent of the adult population suffers from varicose veins.

The heart pumps oxygenated blood from the lungs through the arteries to the cells throughout the body. Oxygen exchange takes place and blood returns up to the heart through the veins. Tiny one-way valves inside our veins function as trapdoors opening and closing with each muscle contraction to prevent the backflow of blood.

Varicose veins result from damage to the valves or vein walls, leading to pooling of blood, vein swelling, and increased venous pressure. Spider veins are a similar condition that affects the smaller veins.

- Cramping

- Fatigue

- Heavy aching in the legs

- Itching and burning

- Swelling of leg or ankle

- Tenderness and pain

If left untreated, varicose veins can worsen over time and cause skin changes such as rash, redness, and sores.

Varicose veins are not life threatening; however, they can increase the risk of developing a blood clot, which is a serious concern. 
- Age: Most common in those over 50 years (but can occur earlier)

- Diet: A low-fibre diet and constipation can contribute to varicose veins.

- Family history

- Gender: Women are affected approximately four times more often than men.

- Lifestyle/occupation: Prolonged sitting or standing with little activity causes pooling of blood and damage to the valves.

- Obesity: Weight gain can increase venous pressure and cause the veins to enlarge.

- Pregnancy: Hormonal changes can weaken vein walls; weight gain increases venous pressure; veins enlarge from increased blood volume; and the growing uterus can compress the veins and increase pressure.

Other factors that may contribute to or worsen varicose veins include: exposure to excessive heat, use of birth control pills or estrogen, and wearing high-heeled shoes.

\section{DR.SHUKLA'S NATURAL PRESCRIPTION}

The incidence of varicose veins is due to lifestyle factors: poor diet (low fibre), lack of activity, and obesity.

\section{Dietary Recommendations}

\section{Foods to include}

- A high-fibre diet is essential to prevent constipation and straining. Aim for 25-35 g of fibre daily. The best food sources are fruits, vegetables, whole grains (oats and bran), legumes, nuts, and seeds (flaxseed and sunflower seeds).

- Berries and grapes provide flavonoids, which strengthen the walls of the veins and help circulation.

- Buckwheat contains rutin, a flavonoid that is beneficial for vein health.

- Fish, nuts, and seeds contain essential fatty acids, which help reduce inflammation, prevent clotting, and improve circulation.

\section{Foods to avoid}

- Saturated fats (animal fats) and trans fats (fast food and processed food) can impede circulation in both the veins and arteries, cause free radical damage, and trigger inflammation.

- Reduce sodium intake from foods such as soda pop, chips, crackers, and deli meats, as sodium can increase fluid retention and swelling.

- Minimize caffeine and alcohol, as they are dehydrating and may worsen symptoms.

\section{Lifestyle Suggestions}

- Exercise regularly. Activities that involve movement of the calf muscle will help pump blood back to the heart.

Try walking, cycling, and swimming. 
- Avoid standing or sitting in the same spot for a long time. Move around, flex your ankles, circle your foot, do calf raises, and shift your body weight.

- Lose excess weight, which will reduce pressure on your legs.

- Avoid excessive heat on your legs (sunbathing and hot baths), which can cause the veins to dilate, thus worsening the problem.

- Elevate your legs when sitting and resting. Don't cross your legs, which hamper circulation and can worsen legaching.

- Minimize or avoid wearing high heels and tight shoes, as they impair circulation.

- Don't wear tight clothing, especially around the waist.

- Avoid constipation and straining on the toilet as this can increase vein pressure.

- Don't smoke, as smoking damages the veins and blood vessels, increases blood pressure, and impairs circulation.

\section{Recommended Pugos Nutrition Supplements}

Nutrease, Astashine, Liquimega

\section{Health is wealth \& wealth is Pugos Nutrition}

From Award-Winning Author

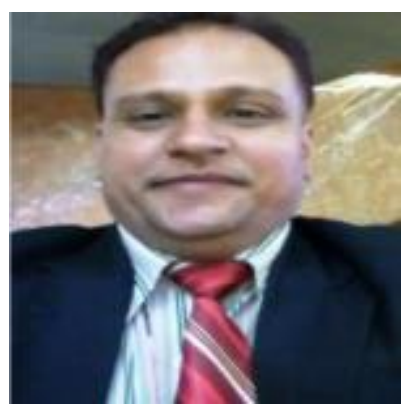

\section{Dr.Govind Shukla, Nutrition Expert}

lactonovaresearch44@gmail.com

Govind Shukla, Specializes in Pharmacology, Toxicology, Nutraceuticals \& Herbal Drugs has published More than 100 research papers in National \& International Journals. He is also a reviewer of the International Journal of Pharmacology \& pharmacotherapeutics, Chief editor of IJPNR Journal \& Freelance Medical Writer for Different publication Groups including Lambert Academic Publishing Saarbrucken, Germany.

\section{Dedication}

Dedicated to my elder brother Mr.Sampath kumar, Managing Director Lactonava Nutripharm Pvt. Ltd., Hyderabad for his uncompromising principles that guided my life and leading into intellectual pursuits. 